\title{
Structure analysis of edge-on spiral galaxies
}

\author{
R. de Grijs and P.C. van der Kruit \\ Kapteyn Astronomical Institute, P.O. Box 800, 9700 AV Groningen, The Netherlands
}

Received July 19; accepted October 17, 1995

\begin{abstract}
The stellar distribution of a small sample of edge-on spiral galaxies is examined in $B, V, R$, and $I$ by fitting model distributions to the light profiles, both perpendicular to the galaxy planes and along the major axes. We have developed a method to compare the fits for the models obtained for different galaxies by introducing a global goodness-of-fit parameter $P_{i j}$. In general, the best fitting vertical model is more peaked than expected for an isothermal sheet distribution, i.e. it is either an exponential light distribution or a $\operatorname{sech}(z)$-model. The most likely explanation for the deviations from an exponential or a $\operatorname{sech}(z)$ distribution at small $z$ is dust extinction, whereas the excess light detected at large $z$ for a few early-type spirals could be due to a thick disk component. The constancy of the exponential scale height of both the thin and the thick disks as a function of position along the major axis is confirmed, although it seems to lose strength in the radial outer parts. It is found that the vertical scale parameters for both the thin and the thick disks are confined within narrow ranges. We show that the scale lengths vary as a function of $z$-distance from the galaxy planes. The smallest scale lengths were measured in the galaxy planes. This can be explained by a combination of absorption effects and a young stellar population, that is supposed to be present in the galaxy planes. The mean ratio of the radial to the vertical scale parameter, $h_{R} / z_{0}$, is $5.9 \pm 0.4$.
\end{abstract}

Key words: galaxies: photometry — galaxies: spiral — galaxies: stellar content — galaxies: structure

\section{Introduction}

Since the introduction of the CCD camera as a highly accurate and linear astronomical measuring device, a number of studies has been devoted to the detailed description of the surface brightness distributions in edge-on spiral galaxies.

The vertical surface brightness distribution of a galaxy and the ratio of vertical to radial scale parameters are fundamental pieces of information needed for studies concerning the formation and evolution of disk galaxies. If the exact surface brightness distribution is known, we can distinguish between several mechanisms, related to the heating of the stellar component in galaxy disks.

\subsection{Theoretical models}

Several models have been proposed and fitted to the surface brightness distributions observed. As is well-known from Freeman's (1970) work, the radial light distribution in the disks of spiral galaxies can be described by an exponentially decreasing luminosity law with increasing galactocentric radius. The simplest model to account for the full three-dimensional surface luminosity distribution of any spiral disk (assuming axial symmetry) is a double ex-

Send offprint requests to: R. de Grijs ponential distribution:

$$
L(R, z)=L_{0} \mathrm{e}^{-z / h_{z}-R / h_{R}},
$$

where $h_{z}$ and $h_{R}$ are the vertical and radial scale parameters, respectively, $z$ is the distance from the galaxy plane, $R$ the position along the major axis, and $L_{0}$ the central surface luminosity in the plane of the galaxy.

Tsikoudi (1977) found, after having studied a sample of large lenticular and spiral galaxies, that the luminosity distribution perpendicular to the galaxy plane in the edge-on galaxies NGC 3115, NGC 4111 and NGC 4762 is well fitted by a steep exponential luminosity decrease, extending over a region of $2^{\prime}$ on each side of the plane. In fact, the exponential behaviour of the vertical profiles of edge-on spirals at large $z$, as found by Tsikoudi (1977), led Freeman (1978) to the observation that this is an expected result of the two-component (bulge and disk) model of galactic structure, providing that the disk is approximately isothermal.

Alternatively, other parametrizations for the vertical luminosity distribution have been suggested. Van der Kruit \& Searle (1981a,b, 1982a,b) have described the vertical distribution of the stars in edge-on spirals by that of an isothermal sheet (the "sech ${ }^{2}$-distribution"), with which they obtained better fits to the inner parts than by using 
an exponential distribution:

$$
L(z)=L_{0} \operatorname{sech}^{2}\left(z / z_{0}\right)
$$

where $z_{0}=2 h_{z}$. At large $z$ the vertical scale parameters are equal for both the exponential and the $\operatorname{sech}^{2}(z)$ model. At intermediate $z$-heights, the most prominent luminosity component in most spiral galaxies is the thin disk, which is thought to be locally isothermal (van der Kruit \& Searle 1981a). However, this isothermal approximation may not hold at very low $z$, because very close to the galaxy plane the thin disk is mixed with younger populations, due to active star formation.

Van der Kruit (1988) has discussed the environmental effects on real disk systems in detail and concludes that near the galaxy plane a deviation from a locally isothermal disk situation could be real (i.e., close to the planes the stars are dynamically cooler than expected theoretically). Therefore he proposed that a more peaked distribution than the isothermal sheet, such as the " $\operatorname{sech}(z)$ distribution", may be a more appropriate one to use:

$$
L(z)=L_{0} \operatorname{sech}\left(z / h_{z}\right) .
$$

Van der Kruit (1988) claims that while the isothermal model will not reproduce Wainscoat's (1986) photometry of the southern edge-on galaxy IC 2531 or Gilmore \& Reid's (1983) star densities towards the South Galactic Pole, these observations would agree with a $\operatorname{sech}(z)$ distribution.

\subsection{The vertical scale parameter}

Another matter of interest that will be studied in this paper is the behaviour of the vertical scale parameter as a function of distance along the major axis. Since the vertical scale height can be related directly to the vertical velocity dispersion in the disks, we may be able to constrain models that describe the dynamical heating mechanisms of galaxy disks.

Van der Kruit \& Searle (1981a,b, 1982a,b) found, at least for their sample of edge-on spirals, that the vertical scale parameter, $h_{z}$, is in good approximation independent of position along the major axis. In later studies, this result has been confirmed by a number of authors, e.g. Kylafis \& Bahcall (1987) and Barnaby \& Thronson Jr. (1992) for the disks of NGC 891 and NGC 5907, respectively. A similar conclusion was reached by Jensen \& Thuan (1982) for NGC 4565. Shaw \& Gilmore (1990) studied a sample of 10 spiral and lenticular edge-on galaxies and found that the variation of disk scale heights as a function of radius is typically within $\pm 3 \%$ of the derived mean value, with no evidence of a dependence on colour or model type adopted. In some of their sample galaxies they concluded that the best-fitting model is a combination of two disk components. The scale height was found to be constant at the same $3 \%$ level in both disk components.
It should be noted that these results were obtained from two-dimensional modeling.

\subsection{Models for the dust distribution}

In general, it is assumed that the influence of dust extinction is largest in the planes of galaxies. In fact, observations of edge-on galaxies reveal strong dust lanes near the galaxy planes in many cases. Numerous studies have been devoted to the description of the dust influence in optical wavelengths, e.g. Disney et al. (1989, hereafter DDP) and Kylafis \& Bahcall (1987, hereafter KB87).

According to DDP, the so-called "sandwich model" is the most physically attractive model for the description of the dust distribution: it is believed that in general, through dissipation, dust and gas will sink into an obscuring layer (containing a mix of absorbing and emitting material) narrower than the emitting layer. This is observed in our Galaxy as well as in external edge-on galaxies (e.g. KB87). In our Galaxy, for instance, we can distinguish between the local (dynamically) cool gas layer with a scale height of 135 pc (e.g. Cowie \& Songaila 1986), opposed to a scale height of 325 pc for the stars (Gilmore \& Reid 1983). As is obvious from geometrical considerations, if the distribution of dust, gas, and stars in a galaxy is best represented by a sandwich model, some stars will be partly obscured while others will be in front of the obscuring layer, leading to the appearance of an optically thin disk on top of an optically thick body. Thus, according to DDP a sandwich model can represent any disk in which the obscuring characteristics vary from point to point (like a vertically-layered disk with an upper crust of unobscured stars, a disk with obscuring patches, a disk in which the optical depth falls off as a function of radius, or a combination of these).

\subsection{The ratio of radial to vertical scale parameters}

Since we are looking at edge-on galaxies, we can easily measure the ratio of the radial to the vertical scale parameter for galaxy disks, $h_{R} / z_{0}$.

By studying this ratio statistically, we may be able to put constraints on the physical processes that formed the disks as well as on the stability parameters of these systems. The scale height is presumably determined by secular evolution of the stellar velocity dispersion, and statistics on the ratio of scalelength to scale height gives information on the importance of this process in galaxies with different properties, like radial scalelength, integrated magnitude, and absolute luminosity.

Bottema (1993) has shown that a constant value for the $h_{R} / z_{0}$ ratio leads to a more or less constant mass-tolight ratio of the old disk, $(M / L)_{B}$, under the assumptions that we are dealing with exponential, locally isothermal disks with a constant ratio of vertical to radial velocity 
dispersion. On the other hand, if we assume a linear relationship between the old-disk absolute luminosity and the vertical velocity dispersion, Bottema (1993) shows that (for a constant $\left.(M / L)_{B}\right)$ the ratio $h_{R} / z_{0}$ decreases rapidly from faint galaxies to a constant level for normal and bright galaxies.

From a kinematical point of view, this $h_{R} / z_{0}$ ratio is also important, since once this ratio is known, we can determine the maximum rotation of a disk from measurements of the vertical disk dispersion (Bottema 1993). In order to fit the observations of the maximum rotational velocity of disk-dominated Sc galaxies, $h_{R} / z_{0}$ needs to be on order 10. However, van der Kruit \& Searle (1982a) found a $h_{R} / z_{0}$ ratio of $4.7 \pm 1.8$ (in the optical $J$ band) for a number of galaxies comparable to our own Galaxy. This leads Bottema (1993) to the conclusion that, for realistic $h_{R} / z_{0}$ values, the stellar velocity dispersions only allow the disk to have a (theoretical) maximum rotation of on average $63 \%$ of the observed maximum rotation. This is significantly less than results of the so-called maximum disk fits that are widely used to model rotation curves.

In this paper we present broad-band optical observations and model fits of a small sample of edge-on spiral galaxies. The observations presented here form part of a project intended to study the structural parameters of edge-on spiral galaxies statistically. In Sect. 2 we will give an overview of the observations and a brief description of the reduction techniques used. In Sect. 3 we will first present the results of this study, of which the implications will be discussed in Sect. 4 .

\section{Observations and data reduction}

\subsection{Sample selection and observations}

For a statistical study of the structural parameters of edgeon spiral galaxies we selected a sample on basis of the Uppsala General Catalog (UGC, Nilson 1973) with the following properties:

- their inclinations are greater than or equal to $87^{\circ}$;

- the angular blue diameters $\left(D_{25}\right)$ are larger than 2.2 ;

- the galaxy types are ranging from S0 to Sd, and

- they should be non-interacting.

The inclinations were determined following Guthrie (1992), assuming a true axial ratio $\log R_{0}=0.95 \pm 0.03$, corresponding to an intrinsic flattening $q_{0}=(b / a)_{0}$ of 0.11 \pm 0.01 . From this intrinsic flattening the inclinations $i$ were derived by using Hubble's (1926) formula

$$
\cos ^{2} i=\left(q^{2}-q_{0}^{2}\right) /\left(1-q_{0}^{2}\right),
$$

where $q=b / a$ is the observed axis ratio. The inclination of UGC 8286 was determined by comparing the positions of $\mathrm{H}$ II regions with the symmetry plane of the galaxy.

Imaging CCD observations of 8 edge-on galaxies in $B$, $V, R$, and $I$ were obtained with the $1 \mathrm{~m}$ Jacobus Kapteyn
Telescope (JKT) of the Observatorio del Roque de los Muchachos at La Palma (Spain), equipped with the EEV7 CCD. The EEV7 CCD, with $1280 \times 1180$ pixels and a pixel size of 0 "' 30 , was used in slow read-out mode, in this way reducing the read-out noise to below the sky noise. We used the $B, V$ and $R$ Harris filters and the Kitt Peak $I$ filter available on La Palma (RGO/La Palma Technical Notes 1987, 1990).

Details of the specific observations can be found in Table 1; I-band images of the sample galaxies are presented in Fig. 1.

\subsection{Reduction}

The standard photometric reduction was applied to the CCD images by using the ESO-MIDAS data reduction package, developed by the European Southern Observatory. In order to reduce the influence of detector noise, we combined twilight flat fields from several nights to create a very accurate flat field for each passband. The variations in flat field structures from night to night were less than $0.5 \%$ of the total intensity of the individual flat fields. After the flatfielding the background was set to zero so that only the contributions of the galaxies themselves remained. Possible gradients in the background were removed by a background fitting routine, which fitted a twodimensional polynomial (linear in both dimensions) to regions far away from the galaxy and subtracted this twodimensional plane from the image; the remaining background variations are statistical: the uncertainties are mainly due to Poisson noise.

For some of the sample galaxies the seeing is rather bad (i.e. larger than $2^{\prime \prime}$ ). However, this does not influence the results presented in this paper significantly, because the structures looked at extend over many seeing cells and thus the slopes of the surface brightness profiles are not affected significantly.

The calibration of the pixel size was done by using 5 stars in a few object frames and supplementing them with the coordinates from the Guide Star Catalog (Russell et al. 1990). The average measured pixel size was $0{ }^{\prime \prime} 298 \pm 00^{\prime \prime} 004$, not depending on filter, and therefore a pixel size of $0 . ' 30$ was used in the reduction of the observations.

The science frames were flux calibrated by using the standard field PG 0918+028 and the standard stars HD 84971 and 107-Z-544 (Landolt 1983, 1992). These frames were calibrated to Johnson $B$ and $V$ and Kron-Cousins $R$ and $I$. On the photometric nights, observations of (at least five) standards at different airmasses were taken, so that the influence of atmospheric extinction could be corrected for as well.

Vertical profiles were extracted at a number of positions along the major axes of the galaxies. In order to retain reasonable signal-to-noise $(S / N)$ ratios out to large $z$-heights, we applied an intensity-weighted binning algorithm to the vertical profiles from those $z$-heights outwards 
Table 1. Observational data. Meaning of the columns: (1) UGC name; (2) Revised Hubble index (from the RC3); (3) inclination; (4) distance to the galaxy, using either cluster membership or velocities determined from radio observations and a Hubble constant $H_{0}=100 \mathrm{~km} \mathrm{~s}^{-1} \mathrm{Mpc}^{-1}$; (5) passband used; (6) and (7) exposure time and date of observations; (8) seeing conditions during the observations; (9) major and minor axis (and minor axis including bulge) at the $\mu_{B}=25, \mu_{V}=25, \mu_{R}=24$, and $\mu_{I}=23.5 \mathrm{mag} \operatorname{arcsec}^{-2}$ isophote, measured in this study; and (10) comparison with major and minor axis measurements from the literature ${ }^{(4)}$

\begin{tabular}{|c|c|c|c|c|c|c|c|c|c|}
\hline$\overline{\text { Galaxy }}$ & $T$ & $i\left(^{\circ}\right)$ & $\begin{array}{l}\text { Dist. } \\
\text { (Mpc) }\end{array}$ & band & $\begin{array}{l}\text { Exp. time } \\
\text { (sec) }\end{array}$ & $\begin{array}{c}\text { Date of } \\
\text { Observation }\end{array}$ & $\begin{array}{c}\text { Seeing } \\
\left({ }^{\prime \prime}\right)\end{array}$ & $\begin{array}{r}a \times b\left(b_{\text {bulge }}\right) \\
(\operatorname{arcmin})^{(1)}\end{array}$ & $\begin{array}{l}D_{25} \times d_{25} \\
(\operatorname{arcmin})^{(4)}\end{array}$ \\
\hline (1) & (2) & (3) & (4) & (5) & (6) & (7) & (8) & (9) & $(10)$ \\
\hline \multirow{4}{*}{ UGC 7617} & $7.0 \pm 0.9$ & 89 & 11.0 & $B$ & 3600 & $23 / 04 / 93$ & 3.0 & $2.52 \times 0.33$ & $2.41 \times 0.22^{a}$ \\
\hline & & & & $V$ & 2100 & $23 / 04 / 93$ & 2.6 & $2.71 \times 0.54$ & \\
\hline & & & & $R$ & 900 & $23 / 04 / 93$ & 1.9 & $2.37 \times 0.34$ & \\
\hline & & & & $I$ & 1800 & $23 / 04 / 93$ & 2.1 & $2.71 \times 0.50$ & \\
\hline \multirow[t]{3}{*}{ UGC 7808} & $3.0 \pm 1.2$ & 90 & 11.0 & $B$ & 3600 & $22 / 04 / 93$ & 1.6 & $3.38 \times 0.22(0.48)$ & $2.5 \times 0.3:^{b}$ \\
\hline & & & & $V$ & 2100 & $22 / 04 / 93$ & 1.6 & $3.46 \times 0.32(0.54)$ & \\
\hline & & & & $I$ & 847.9 & $21 / 04 / 93$ & 3.3 & $3.53 \times 0.22(0.47)$ & $3.5 \times 0.4^{b, c}$ \\
\hline \multirow[t]{5}{*}{ UGC 7980} & $-1.0 \pm 0.6$ & 90 & 10.7 & $B$ & 1800 & $22 / 04 / 93$ & 1.4 & $4.68 \times 1.08(1.21)$ & $4.5 \times 1.3^{b}$ \\
\hline & & & & $B$ & 1800 & $23 / 04 / 93$ & 2.6 & $4.68 \times 1.08(1.21)$ & \\
\hline & & & & $V$ & 600 & $22 / 04 / 93$ & 1.4 & $4.86 \times 1.37(1.49)$ & \\
\hline & & & & $R$ & 900 & $20 / 04 / 93$ & 1.9 & $5.2 \times 1.6^{(3)}$ & \\
\hline & & & & $I$ & 900 & $24 / 04 / 93$ & 2.4 & $4.62 \times 1.24(1.38)^{(2)}$ & $4.3 \times 1.3^{b}$ \\
\hline \multirow[t]{4}{*}{ UGC 8016} & $-2.0 \pm 0.3$ & 90 & 13.5 & $B$ & 5400 & $04 / 03 / 94$ & 1.8 & $5.65 \times 1.31$ & $9.0 \times 2.0^{b}$ \\
\hline & & & & $V$ & 2100 & $22 / 04 / 93$ & 1.4 & $5.70 \times 1.52$ & \\
\hline & & & & $R$ & 1200 & $22 / 04 / 93$ & 1.4 & $5.42 \times 1.22^{(2)}$ & \\
\hline & & & & $I$ & 1200 & $22 / 04 / 93$ & 1.4 & $5.26 \times 1.31^{(2)}$ & $9.5 \times 2.0^{b}$ \\
\hline \multirow[t]{3}{*}{ UGC 8286} & $5.5 \pm 0.7$ & 88.3 & 4.8 & $B$ & 3600 & $24 / 04 / 93$ & 2.6 & $6.22 \times 0.77$ & $6.5 \times 1.0^{d}$ \\
\hline & & \pm 0.5 & & $V$ & 2100 & $24 / 04 / 93$ & 2.8 & $6.38 \times 0.92$ & \\
\hline & & & & $I$ & 1800 & $24 / 04 / 93$ & 2.6 & $5.72 \times 0.75$ & \\
\hline \multirow[t]{3}{*}{ UGC 8935} & $-2.0 \pm 0.4$ & 90 & 40.4 & $B$ & 3600 & $24 / 04 / 93$ & 1.9 & $3.30 \times 0.53(0.67)$ & $3.9 \times 0.7^{e}$ \\
\hline & & & & $V$ & 2100 & $24 / 04 / 93$ & 2.4 & $3.45 \times 0.69(0.78)$ & \\
\hline & & & & $I$ & 900 & $24 / 04 / 93$ & 2.1 & $3.51 \times 0.63(0.74)$ & \\
\hline \multirow[t]{3}{*}{ UGC 9127} & $5.0 \pm 0.5$ & 87 & 29.6 & $B$ & 3600 & $22 / 04 / 93$ & 1.6 & $6.24 \times 0.78$ & $6.2 \times 0.7^{b}$ \\
\hline & & & & $V$ & 600 & $22 / 04 / 93$ & 1.9 & $6.12 \times 0.72$ & \\
\hline & & & & $I$ & 900 & $20 / 04 / 93$ & 3.0 & $6.0 \times 0.7^{(3)}$ & $6.0 \times 0.6^{b}$ \\
\hline \multirow[t]{3}{*}{ UGC 10043} & $4.0 \pm 0.9$ & 90 & 22.7 & $B$ & 3600 & $03 / 03 / 94$ & 2.3 & $2.13 \times 0.17(0.35)$ & $2.4 \times 0.3^{b}$ \\
\hline & & & & $V$ & 2000 & $03 / 03 / 94$ & 2.4 & $2.31 \times 0.21(0.45)$ & \\
\hline & & & & $I$ & 900 & $23 / 04 / 93$ & 2.1 & $2.57 \times 0.29(0.57)$ & $2.2 \times 0.3^{b}$ \\
\hline
\end{tabular}

(1) - The errors in the values for $a \times b$ are typically 0 ! $17 \times 0$ ! 10 ;

(2) - For UGC 7980 and UGC 8016 the $I$ sizes tabulated are the sizes at $\mu_{I}=22.5$ mag $\operatorname{arcsec}^{-2}$; the $R$ sizes tabulated for UGC 8016 are the sizes at $\mu_{R}=23 \mathrm{mag} \operatorname{arcsec}^{-2}$.

(3) - These sizes are upper limits, since no accurate calibration was available for the data obtained on 20/04/1993.

(4) - Literature used:

${ }^{a}$ Guthrie B.N.G., 1992, A\&AS 93, 255

${ }^{b}$ Hummel E., Beck R., Dettmar R.-J., 1991, A\&AS 87, 309

${ }^{c}$ Haynes M.P., Giovanelli R., 1986, ApJ 306, 466

${ }^{d}$ Staveley-Smith L., Davies R.D., 1987, MNRAS 231, 833

$e$ Vaucouleurs G. de, Vaucouleurs A. de, Corwin H.G. Jr., Buta R.J., Paturel G., Fouqué P., 1991. Springer-Verlag, New York (Third Reference Catalogue)

where the intensities dropped below 4 times the sky noise. In that way, the galaxy profiles could be followed further out.

In the radial direction the galaxies were also binned to retain an approximately constant overall $S / N$ ratio in the vertical profiles.

The initial $S / N$ ratio was determined by the vertical profiles taken at the galaxy centers; we determined the positions of the galaxy centers by fitting ellipses to the galaxies at the lowest isophotal levels. The central areas were left out of the the fitting, because there the various el- lipse fitting routines break down due to, e.g., non-elliptical isophotes caused by the presence of a dust lane.

The positions of the galaxy planes were determined by folding the vertical profiles and under the assumption of symmetrical light distributions (in the case of perfectly edge-on orientations) with respect to the planes.

In some of the sample galaxies the central areas are dominated by the bulge light contribution. Therefore, we applied a bulge-disk decomposition in the radial direction to all sample galaxies. In the following, those vertical profiles that were taken at radii along the major axis where 
the bulge influence is less than $5 \%$ of the total light contribution will be called "disk profiles".

To study the structure in the radial direction, we extracted vertically integrated radial profiles for all galaxies. From these profiles, both the reliability of the background subtraction algorithm and the radial scale parameters of the galaxies could be examined. Secondly, we extracted radial profiles of $5^{\prime \prime}$ width parallel to the major axes of the galaxies. These profiles were used for the study of the dependence of scalelength as a function of $z$-height above the galaxy planes and thus the study of the geometry of the dust component in the sample galaxies, if present.

To be sure that the position angles of the galaxies were determined with sufficient precision an ellipse fitting program was applied to the individual observations, yielding position angle distributions as a function of position along the major axes. From these distributions the best-fitting position angles were determined, with an accuracy of better than $0.5^{\circ}$. Position angles resulting from fits to both the inner and the outer parts of the galaxies were ignored, because of possible contamination from bulge light in the inner parts and distortions due to warps in the outer parts. The best position angles determined for the sample galaxies are listed in Table 2. The position angles listed in Table 2 were corrected for the slight misalignment from the north-to-south direction of the CCD. The misalignment angle for the April 1993 observing run was measured to be $(0.68 \pm 0.02)^{\circ}$.

\section{Results}

\subsection{Vertical surface brightness distributions}

\subsubsection{Exponential scale heights}

From the individual vertical profiles the exponential scale height behaviour as a function of position along the major axis was studied for each of the sample galaxies.

The simplest way to do this is by fitting an exponential light distribution to the intermediate $z$-heights of the vertical profiles. In order to minimize the influence of a possible dust lane or a second, underlying disk component (we will call such components "thick disks"), the exponential fits were done in these (intermediate) regions on both sides of the galaxy planes. The extents of the regions where we fitted exponential light distributions are tabulated in Table 2. For each galaxy we used the same region for all passbands; results of the fitting are listed in Table 4.

We have examined the influence of the choice of the fitting boundaries. Especially in the (later type) dusty galaxies, the effect of varying the inner fitting boundary can be considerable. In order to estimate the importance of accurately defining the fitting boundaries, we have looked at the differences that arise when fitting the entire vertical range compared to the fit between the fixed boundaries as has been done in this paper. The maximum difference between these two methods can amount up to $\sim 30 \%$ ( $B$ band) in the dustiest galaxy (UGC 9127). However, our estimate of the realistic errors in the exponential scale height determination caused by a different definition of the fitting boundaries is on average $\sim 8 \%$ in $B$, decreasing to $\sim 4 \%$ in $I$, with an extra scatter of a few per cent introduced by the dustiness of the galaxy. The influence of a narrow dust lane, like in UGC 7980, is insignificant in this respect.

Although the resulting exponential scale heights may vary considerably depending on the fitting boundaries chosen, the ratios of the scale heights determined in different passbands remain more or less constant. In Table 3 we have, as an example, tabulated the effects of choosing different fitting boundaries for UGC 9127, from taking the entire vertical luminosity profile, including the prominent dust lane (fitting range from 0 to $30^{\prime \prime}$ ), to taking a low $S / N$ region in the (vertically) outer parts of the galaxy (region from 15 to $25^{\prime \prime}$ ). It can be seen in this table that the ratios of the scale heights between the various passbands only vary by $\sim 3-4 \%$ when choosing slightly different fitting boundaries in the intermediate part of the vertical luminosity distribution, i.e. the regions from $5-25^{\prime \prime}$ or from $10-20^{\prime \prime}$. UGC 9127 was taken because of its very prominent dust lane, which might influence the results obtained significantly.

Before we describe the results of the model-fitting, it is necessary to study the effect of a possible non-edge-on view at the galaxy planes. Van der Kruit \& Searle (1981a) showed that for systems having an inclination $i \geq 86^{\circ}$ the slopes of both the vertical and radial profiles remain unaltered. The inclinations of the galaxies that are studied in this paper range from 87 to $90^{\circ}$, so that the results presented here will not significantly be affected by inclination effects.

In Fig. 2 the behaviour of the exponential scale height as a function of position along the major axis is shown for all sample galaxies and all passbands used. In this figure we did not correct for the influence of bulge light contributions to the vertical luminosity profiles. In Table 2 the positions along the major axes of the bulge-dominated profiles are tabulated.

Especially in the later-type galaxies, the exponential disk scale height was found to be constant as a function of position along the major axis, in our case the constancy of the disk scale height was found to be of order 10 to $15 \%$ in the Sc and later-type galaxies. This constancy level differs significantly from those found by Shaw \& Gilmore (1989). However, they tried to model their sample galaxies globally by applying a two-dimensional model to their data, whereas we have fitted one-dimensional models to different parts of our sample galaxies. Our results give more direct information about the irregularities on small scales.

However, in most of the galaxies the fitting of exponential light distributions indicates smaller slopes and hence larger values for $h_{z}$ in the (radial) outer parts than in 

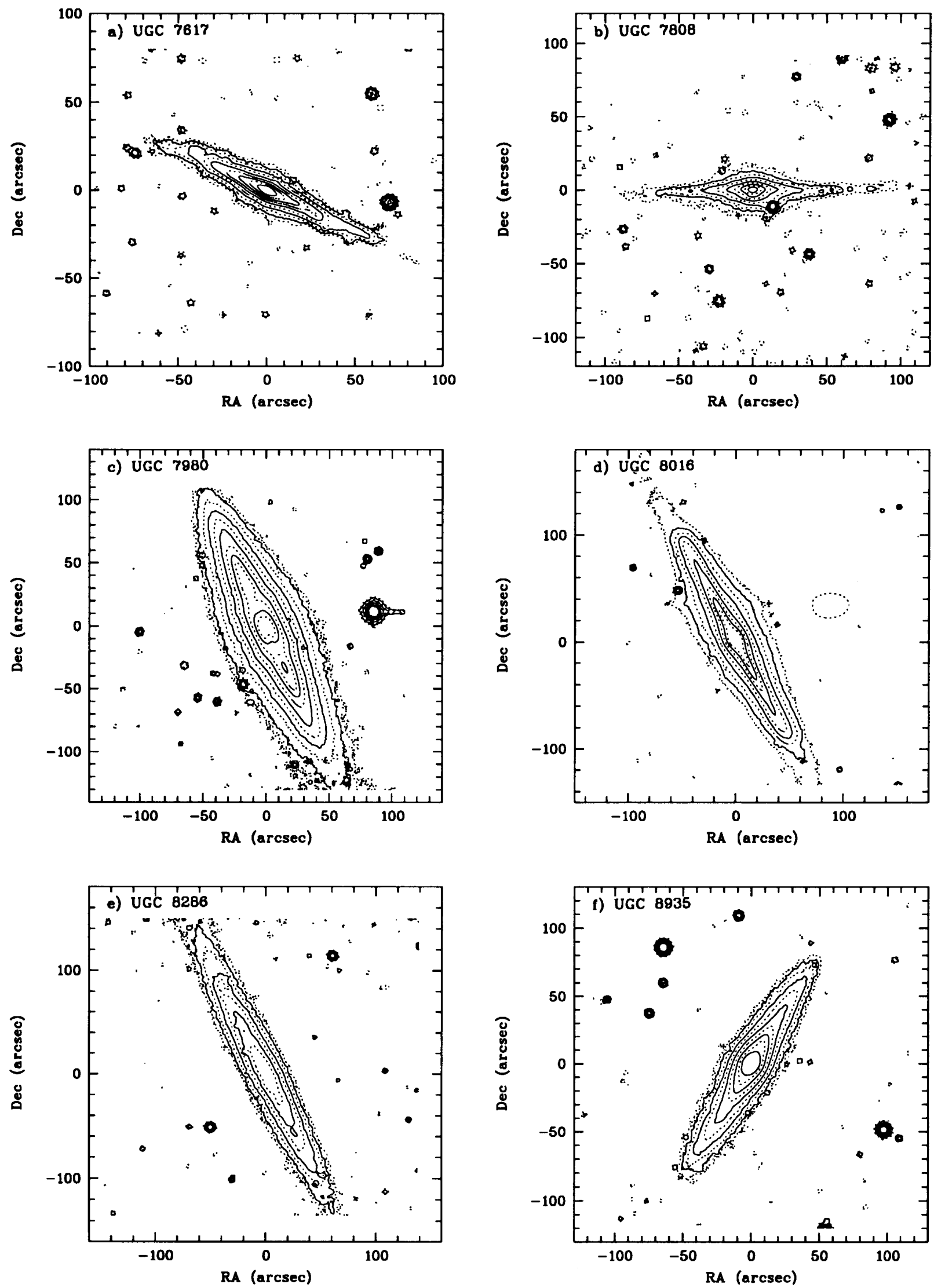

Fig. 1. $I$-band images of the sample galaxies. The lowest contour level is $23.5 I$-mag $\operatorname{arcsec}^{-2}$, except for UGC 7980 and UGC 8016 (21.5 I-mag $\left.\operatorname{arcsec}^{-2}\right)$; the difference between subsequent contours is $0.5 \mathrm{I}$-mag $\operatorname{arcsec}^{-2}$. North is at the top, East to the left, instrumental position angles have not been corrected for 

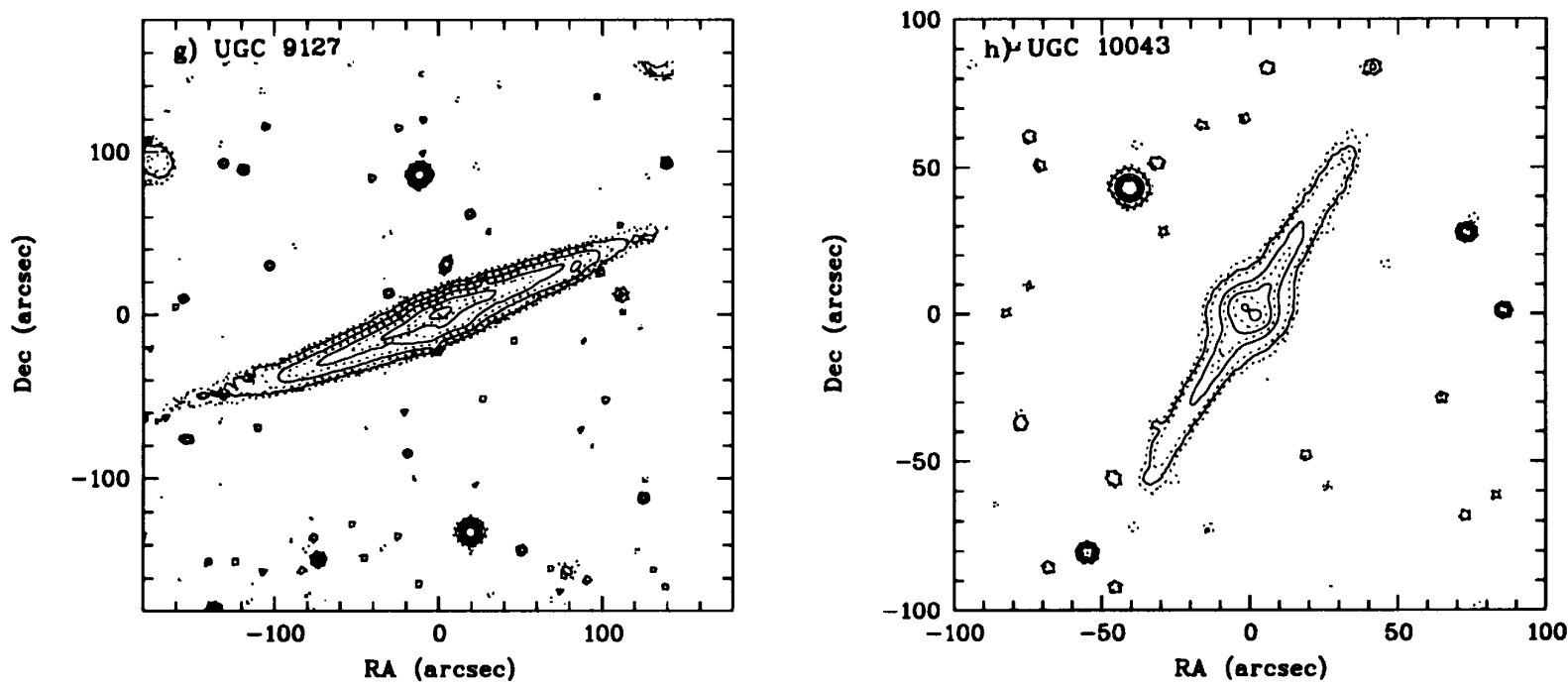

Fig. 1. continued

Table 2. Regions used for the fitting of the exponential scale parameters

\begin{tabular}{|c|c|c|c|c|c|c|c|}
\hline \multirow[t]{2}{*}{$\overline{\text { Galaxy }}$} & \multicolumn{2}{|c|}{ Region of vertical fit } & \multirow{2}{*}{$\begin{array}{c}\text { Bulge-dominated } \\
\text { major axis positions } \\
\left(h^{-1} \mathrm{kpc}\right)\end{array}$} & \multicolumn{2}{|c|}{ Region of radial fit } & \multicolumn{2}{|c|}{$\mathrm{PA}\left({ }^{\circ}\right)^{(1)}$} \\
\hline & $\left({ }^{\prime \prime}\right)$ & $\left(h^{-1} \mathrm{kpc}\right)$ & & $\left({ }^{\prime \prime}\right)$ & $\left(h^{-1} \mathrm{kpc}\right)$ & (measured) & (RC3) \\
\hline UGC 7617 & $5-15$ & $0.27-0.80$ & - & $10-45$ & $0.54-2.40$ & 64.5 & 68 \\
\hline UGC 7808 & $5-10$ & $0.27-0.53$ & $-0.31-0.23$ & $20-60$ & $1.07-3.20$ & 91.7 & 95 \\
\hline UGC 7980 & $10-30$ & $0.52-1.56$ & $-1.4-1.5$ & $40-80$ & $2.08-4.15$ & 24.9 & 27 \\
\hline UGC 8016 & $20-40$ & $1.31-2.62$ & $-0.5-0.5$ & $40-120$ & $2.62-7.86$ & 28.3 & 32 \\
\hline UGC 8286 & $10-25$ & $0.23-0.58$ & - & $40-100$ & $0.93-2.32$ & 26.5 & 28 \\
\hline UGC 8935 & $5-15$ & $0.98-2.94$ & $-3.3-3.3$ & $20-50$ & $3.92-9.79$ & 151.5 & 152 \\
\hline UGC $9127^{(2)}$ & $10-20$ & $1.44-2.88$ & $-1.5-1.5$ & $30-90$ & $4.31-12.9$ & 113.4 & 115 \\
\hline UGC 10043 & $8-15$ & $0.80-1.65$ & $-1.5-1.5$ & $15-40$ & $1.65-4.40$ & 149.5 & 151 \\
\hline
\end{tabular}

(1) The position angles listed here are those measured and taken from the Third Reference Catalogue (RC3, De Vaucouleurs et al. 1991), respectively.

(2) Due to a prominent dust lane we only fitted the light profiles south of the plane.

Table 3. Effects of choosing different boundaries for the fitting of the exponential scale heights for UGC 9127

\begin{tabular}{|c|c|c|c|c|c|c|}
\hline \multirow{2}{*}{$\begin{array}{c}\text { Fitting range } \\
\left({ }^{\prime \prime}\right)\end{array}$} & \multicolumn{3}{|c|}{ Exponential scale heights $\left(h^{-1} \mathrm{kpc}\right)$} & \multicolumn{3}{|c|}{ Scale height ratios } \\
\hline & $B$ & $V$ & $I$ & $B / I$ & $B / V$ & $V / I$ \\
\hline $0-30$ & $0.96 \pm 0.22$ & $0.86 \pm 0.06$ & $0.86 \pm 0.17$ & 1.11 & 1.11 & 1.00 \\
\hline & & & 0 & & 0 . & 1.25 \\
\hline $10-20$ & $0.63 \pm 0$ & $0.68 \pm 0.08$ & $0.58 \pm 0$ & 1.09 & 0.92 & 1.17 \\
\hline $15-25$ & $0.85 \pm 0.20$ & _(1) & $0.68 \pm 0.11$ & 1.26 & - & - \\
\hline
\end{tabular}

(1) No data available due to too few data points in this region.

The range from $10-20^{\prime \prime}$ was used for the determination of the exponential scale height in Table 4 .

the central areas of the disk. Although our data is of sufficiently high quality to detect small variations in scale height, we need deeper observations to be able to quantify the radial increase of the scale heights in these later-type galaxies.

In the early-type galaxies UGC 7980, UGC 8016 and UGC 8935, this increase is much more evident and is obviously influenced by the bulge light contribution. In UGC 7808 and UGC 10043, the exponential disk scale heights in the central regions are clearly also affected by the bulge light contribution.

The radial increase of the disk exponential scale height in UGC 9127 is due to a clear warp in the stellar body. We will come back to this in Sect. 4.2, where we will discuss various possible causes for the remarkable constancy of the exponential scale height. Finally, there is no clear trend of scale height variation with passband. We will come back to this in Sect. 4.2 as well. 
Table 4. Global goodness-of-fit parameters obtained for the sample galaxies. Columns: (1) Galaxy name; (2) broad band filter used; (3) Total number of disk profiles; (4-6) global goodness-of-fit parameters for single-component models as described in the text; (7-9) global goodness-of-fit parameters for multi-component fits, compared to the best single-component fit; (11) exponential scale height and its statistical error

\begin{tabular}{|c|c|c|c|c|c|c|c|c|c|}
\hline Galaxy & passband & $N_{\text {disk }}$ & $P_{12}$ & $P_{13}$ & $P_{23}$ & $P_{1+1,1}$ & $P_{2+1,1}$ & $P_{3+1,1}$ & $\begin{array}{c}h_{z}^{\exp } \text { (const) } \\
\text { (in kpc) } \\
(10) \\
\end{array}$ \\
\hline \multirow[t]{4}{*}{ UGC 7617} & $B$ & 8 & 2.411 & 1.454 & 0.603 & - & - & - & $0.10 \pm 0.01$ \\
\hline & $V$ & 8 & 0.895 & 0.573 & 0.640 & - & - & - & $0.11 \pm 0.01$ \\
\hline & $R$ & 8 & 0.551 & 0.344 & 0.624 & - & - & - & $0.11 \pm 0.01$ \\
\hline & $I$ & 8 & 0.891 & 0.574 & 0.644 & - & - & - & $0.11 \pm 0.01$ \\
\hline \multirow[t]{3}{*}{ UGC 7808} & $B$ & 6 & 1.108 & 1.005 & 0.907 & - & - & - & $0.13 \pm 0.01$ \\
\hline & $V$ & 6 & 0.954 & 0.822 & 0.862 & - & - & - & $0.13 \pm 0.02$ \\
\hline & $I$ & 6 & 1.055 & 0.898 & 0.852 & - & - & - & $0.13 \pm 0.01$ \\
\hline \multirow[t]{4}{*}{ UGC $7980^{(1)}$} & $B$ & 27 & 0.235 & 0.148 & 0.630 & 0.182 & 0.231 & 0.250 & $0.46 \pm 0.02$ \\
\hline & $V$ & 32 & 0.208 & 0.129 & 0.623 & 0.317 & 0.461 & 0.367 & $0.40 \pm 0.04$ \\
\hline & $R$ & 22 & 0.182 & 0.111 & 0.610 & 0.111 & 0.205 & 0.205 & $0.46 \pm 0.02$ \\
\hline & $I$ & 17 & 0.166 & 0.095 & 0.571 & 0.265 & 0.249 & 0.236 & $0.43 \pm 0.02$ \\
\hline \multirow[t]{4}{*}{ UGC 8016} & $B$ & 8 & 0.283 & 0.191 & 0.675 & 0.098 & 0.096 & 0.111 & $0.82 \pm 0.07$ \\
\hline & $V$ & 6 & 0.322 & 0.229 & 0.709 & 0.102 & 0.074 & 0.100 & $0.78 \pm 0.07$ \\
\hline & $R$ & 8 & 0.338 & 0.238 & 0.704 & 0.255 & 0.199 & 0.231 & $0.66 \pm 0.09$ \\
\hline & $I$ & 8 & 0.268 & 0.185 & 0.691 & 0.047 & 0.040 & - & $0.64 \pm 0.09$ \\
\hline \multirow[t]{3}{*}{ UGC 8286} & $B$ & 29 & 2.265 & 1.474 & 0.651 & - & - & - & $0.13 \pm 0.01$ \\
\hline & $V$ & 29 & 2.634 & 1.459 & 0.554 & - & - & - & $0.14 \pm 0.01$ \\
\hline & $I$ & 28 & 2.882 & 1.732 & 0.601 & - & - & - & $0.13 \pm 0.02$ \\
\hline \multirow[t]{3}{*}{ UGC 8935} & $B$ & 4 & 1.165 & 0.704 & 0.604 & - & - & - & $0.6 \pm 0.1$ \\
\hline & $V$ & 4 & 0.641 & 0.297 & 0.463 & - & - & - & $0.6 \pm 0.1$ \\
\hline & $I$ & 4 & 0.973 & 0.424 & 0.436 & - & - & - & $0.6 \pm 0.1$ \\
\hline \multirow[t]{3}{*}{ UGC 9127} & $B$ & 16 & 1.387 & 1.371 & 0.988 & - & - & - & $0.63 \pm 0.07$ \\
\hline & $V$ & 16 & 1.388 & 1.353 & 0.975 & - & - & - & $0.68 \pm 0.08$ \\
\hline & $I$ & 14 & 1.220 & 1.134 & 0.930 & - & - & - & $0.58 \pm 0.08$ \\
\hline \multirow[t]{3}{*}{ UGC 10043} & $B$ & 9 & 1.147 & 1.147 & 0.999 & - & - & - & $0.38 \pm 0.04$ \\
\hline & $V$ & 14 & 0.881 & 0.633 & 0.718 & - & - & - & $0.41 \pm 0.03$ \\
\hline & $I$ & 14 & 1.562 & 1.009 & 0.646 & - & - & - & $0.28 \pm 0.01$ \\
\hline
\end{tabular}

(1) Although the two-component fits indicate better results for a double exponential distribution, the combination of a sech and an exponential yields better fits out to larger radii in all passbands.

\subsubsection{More complex modeling of the vertical profiles}

In this section we will try to fit the vertical light profiles, by applying the exponential, the $\operatorname{sech}(z)$ and the $\operatorname{sech}^{2}(z)$ distributions to the observational data.

The fitting routine that was used (Marquardt 1963) is a mixture of the Taylor and the steepest descent methods. It requires as input initial estimates for the values of the vertical scale parameter and the central surface brightness of the individual profiles. In order to yield satisfactory small goodness-of-fit parameters $\left(\chi_{\text {model }}^{2}\right)$, the initial estimates should be of the order of the real physical parameters, although a close match is not necessary. The non-linear least squares fitting stops iterating when successive iterations fail to produce a decrement in the $\chi_{\text {model }}^{2}$ which is less than the minimum possible decrement.

As a first approximation we fitted the total profiles, taking into account all individual data points with the observational errors in each point as the statistical weights. The fitting was done in the logarithmic domain.

Although, in most cases, single-component models give quite satisfactory fits to the data, for some galaxies in the sample (UGC 7980 and UGC 8016 in particular) twocomponent models yield better fits. These two-component models are a combination of one of the three basic models, represented by Eqs. (1)-(3), and a second underlying exponential component.

This underlying thick-disk component shows up only at large $z$-distances, where the three model functions are indistinguishable (see, e.g., van Dokkum et al. 1994). We have chosen to use the exponential model as a representative model for the thick-disk component, because near the plane of the galaxy the influence of the light of this thick disk is negligible compared to the main disk component (i.e., its contribution is less than $1 \%$ of the thin-disk light). Thus, the exact light distribution of the underlying thick disk in the galaxy plane is not important for the present analysis.

\subsubsection{The global goodness-of-fit parameter}

By comparing the values of $\chi_{\text {model }}^{2}$ obtained for each profile, the best-fitting models for all positions along the major axes of the galaxies can be found. However, if we want 

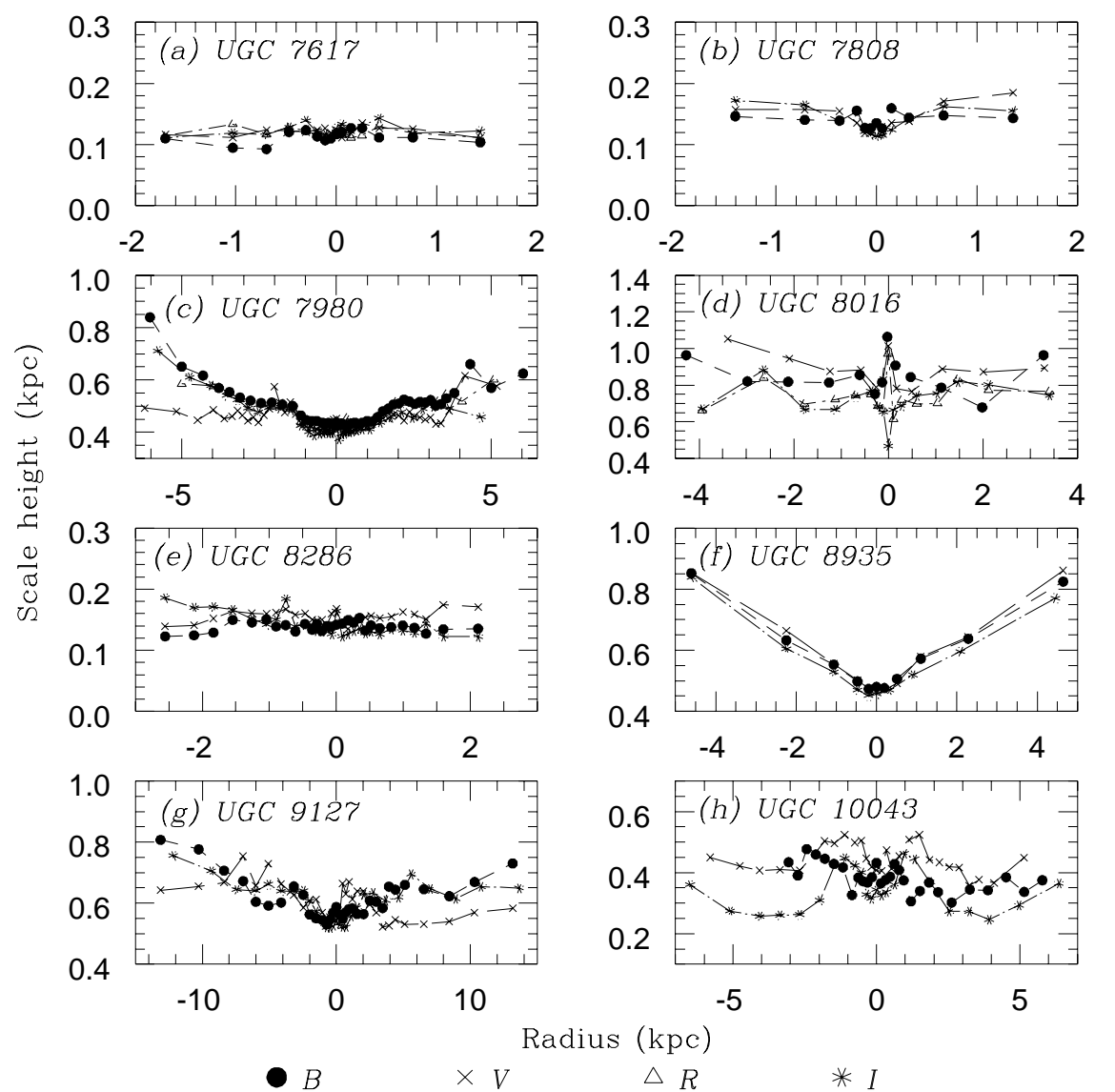

Fig. 2. Exponential scale height distributions for the sample galaxies as a funtion of radius along the major axis; near the galaxy centers the errors are on order of the symbol sizes, increasing up to three times as large in the outer parts. a) UGC 7617, b) UGC 7808, c) UGC 7980, d) UGC 8016, e) UGC 8286, f) UGC 8935, g) UGC 9127, and h) UGC 10043. The bulge-dominated positions can be found in Table 2

to know which model fits the galaxies best in general, we have to define a global goodness-of-fit parameter, $P_{i j}$, where $i$ and $j$ represent the individual models. Only by adopting such an objective statistical measure, one will be able to distinguish between different model combinations without any a priori bias towards a specific model. follows:

The global goodness-of-fit parameter $P_{i j}$ is defined as

$$
P_{i j}=\left(\frac{\sum \chi_{i}^{2}}{\sum \chi_{j}^{2}}\right)_{\mathrm{disk}}
$$

where the sum contains the $\chi^{2}$ 's of all disk-dominated profiles, and the models are represented by numbers $(i, j)$ as $1=\operatorname{exponential;} 2=\operatorname{sech}(z)$; and $3=\operatorname{sech}^{2}(z)$.

In Table 4 the results obtained for the sample galaxies are shown. In the case of multi-component fits, the models were compared with the best-fitting single-component models, which turned out to be exponential light distributions. From the global goodness-of-fit parameters $P_{i j}$ the most representative light distributions were determined, as listed in Table 5.
From Tables 4 and 5, we note that in the later-type galaxies the vertical light distribution is best represented by either an exponential or a $\operatorname{sech}(z)$ single component model. In the early-type galaxies, notably in UGC 7980 and UGC 8016, two-component fits yield better results than single component models. Also in the other earlytype galaxy in our sample, UGC 8935, in a number of vertical profiles the light distribution is best represented by a two-component fit, though the global goodness-offit parameters indicate that single component models are still better in this case. We also tried to fit two-component models to the other galaxies, but we did not detect any other secondary disk components with a scale height significantly different from the main disk component.

It could be argued that the resulting light distributions are significantly influenced by the dust, which is present near the galaxy planes. However, if we redo the modelling starting at the $z$-heights listed in Table 2 (beyond which the influence of dust obscuration has vanished almost completely) the same best-fitting models for the sample galaxies are found. 


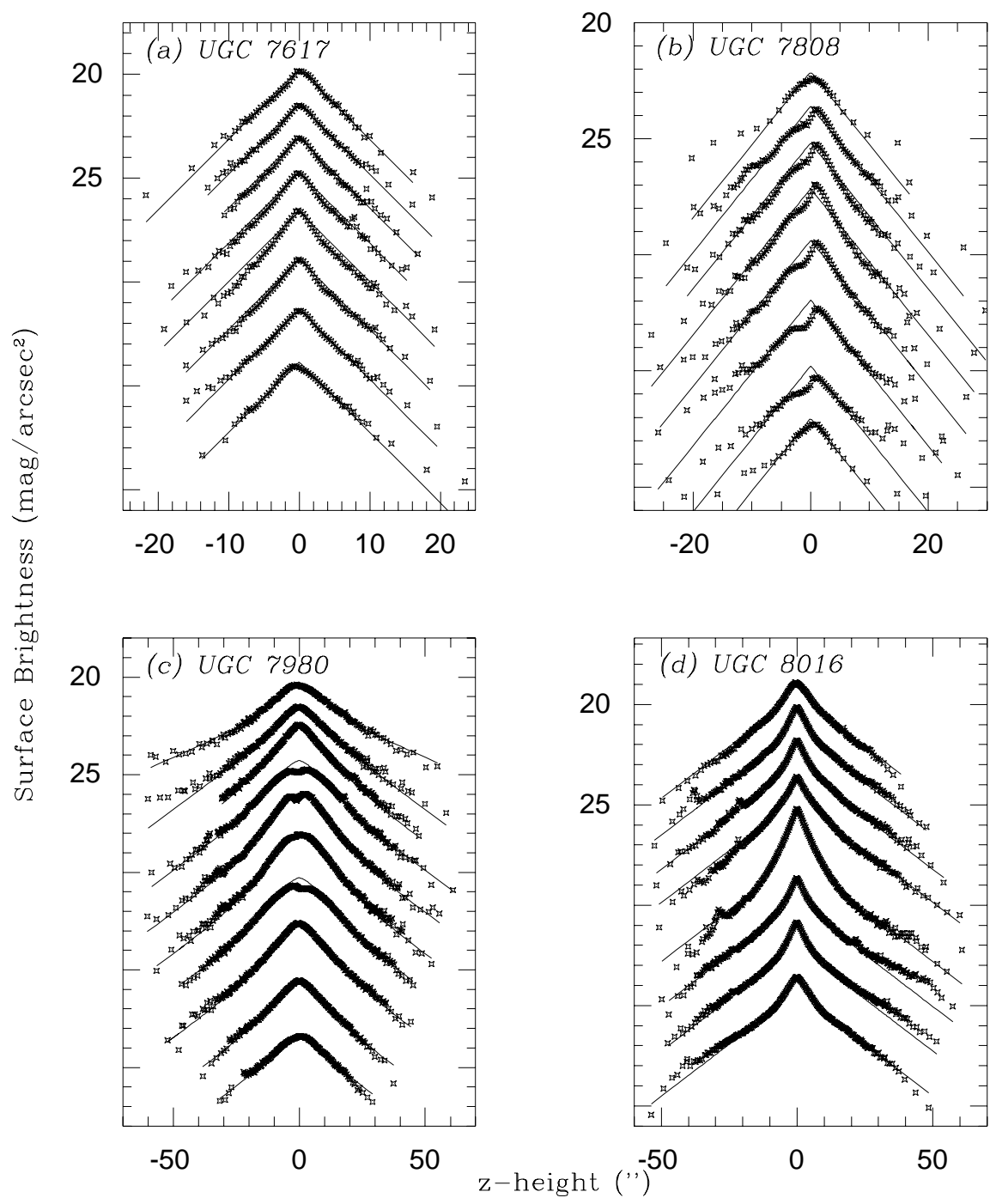

Fig. 3. I-band fits to the vertical profiles for the sample galaxies: a) UGC 7617 , b) UGC 7808 , c) UGC 7980 d) UGC 8016 , e) UGC 8286, f) UGC 8935, g) UGC 9127, and h) UGC 10043. The profiles are offset with respect to the top ones. In Table 6 the positions along the major axes at which the profiles were extracted are listed

In Fig. 3 the fits to the vertical profiles of all sample galaxies are plotted. We only show the $I$-band profiles, because of all bands in which the galaxies were observed the $I$-band profiles are least influenced by dust extinction. Therefore they approximate the true old stellar light distributions best. Since in the $I$ band we are looking at the old(er) disk components, possible thick disk components will also show up clearly, due to their supposedly old(er) ages. It is clear that close to the galaxy planes and near the galaxy centers the fits of pure exponentials or pure $\operatorname{sech}(z)$-functions break down in the case of UGC 7808, UGC 7980, and UGC 9127, due to the presence of an obscuring dust layer. The positions at which the profiles were extracted from the $I$-band images of the galaxies as well as the width of the radial bins are given in Table 6 .

\subsubsection{The vertical scale parameter}

If we compare the values of the exponential scale height $h_{z}^{\exp }$ (Table 4) and the vertical scale parameter $h_{z}$ derived from the fitting of the more complex models (Table 5) for the same passband, we notice the remarkably good agreement between the methods used. Only in the case of two-component best fits the resulting values differ by more than their uncertainties. This shows that in general the determination of the exponential scale heights is a good starting point if one is interested in the behaviour of the vertical scale parameter.

Interesting results in this respect are that the range of vertical scale parameters, as found in the model fitting routines, is quite narrow for both the thin and the thick disk components, as is shown in Fig. 4, and that the scale heights do not vary significantly as a function of passband. 


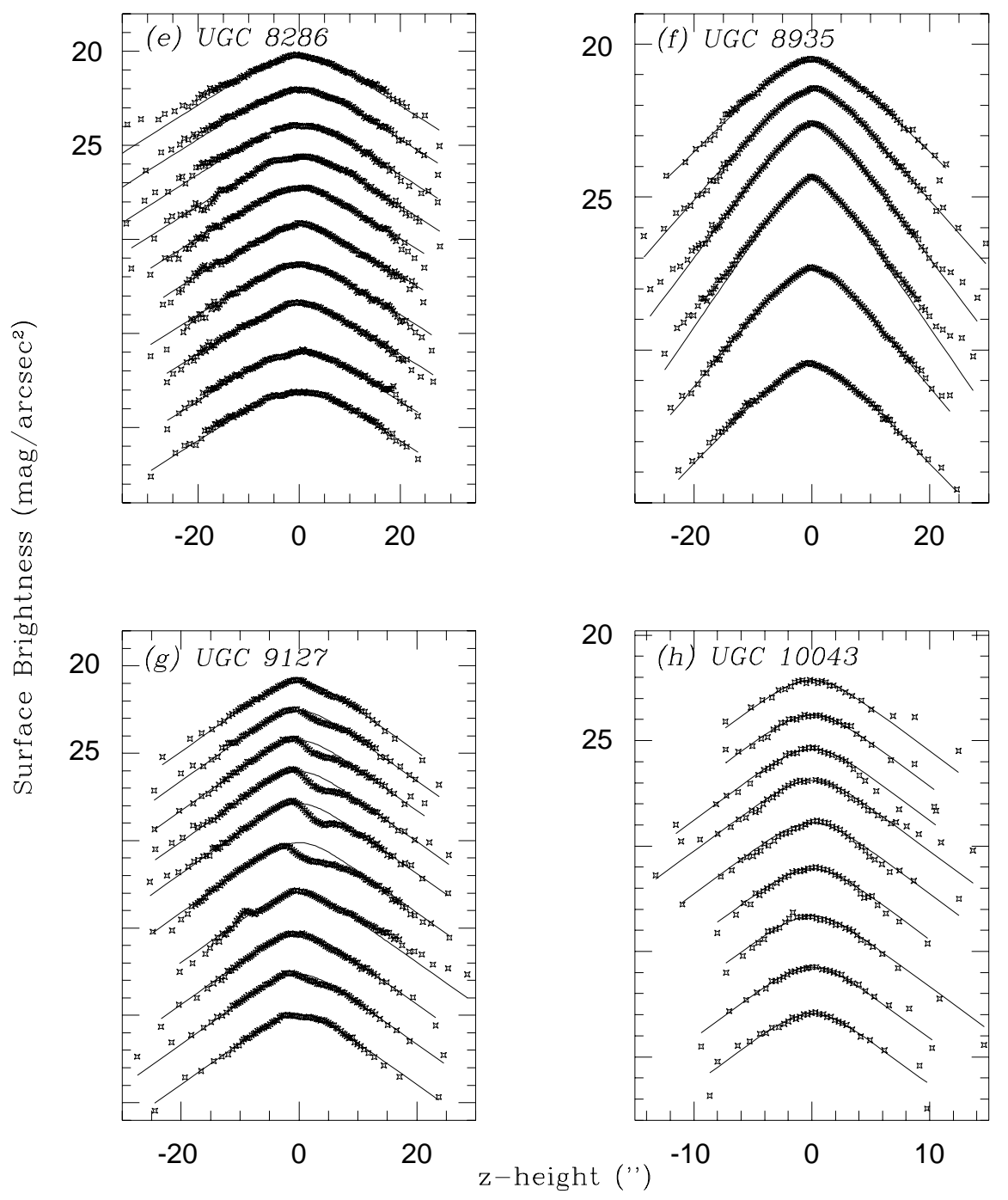

Fig. 3. continued

The vertical scale heights of the thin disks are usually larger than (on order) $100 \mathrm{pc}$ and smaller than $\sim 250 \mathrm{pc}$, except for UGC 8935 and UGC 9127.

In the case of UGC 8935 this can be explained if we assume that the scale height we are fitting is influenced by the presence of a thick disk. If we model the vertical profiles of UGC 8935 under this assumption, the resulting scale parameters are the following. For the thindisk component $h_{z \text {,sech }}(B)=0.3 \pm 0.1 h^{-1} \mathrm{kpc}$ and $h_{z, \text { sech }}(V, I)=0.4 \pm 0.1$ is found, and for the thick-disk component $h_{z, \exp }(B)=0.6 \pm 0.2, h_{z, \exp }(V)=0.7 \pm 0.3$, and $h_{z, \exp }(I)=0.8 \pm 0.3$, where the best-fitting twocomponent model is described by the combination of a $\operatorname{sech}(z)$ and an exponential distribution in all passbands. So the vertical scale parameters of UGC 8935 lie also in the same narrow range (within the uncertainties).

The thick disk scale parameters range from about 470 to 620 pc. From Fig. 4 it turns out that for UGC 9127 the scale height of the single component fitted lies in the range of the thick disk parameters found for the early type galaxies. The explanation for this large thin disk scale height is the dusty appearance of the galaxy, which is probably optically thick (even in the $I$ band) out to about two scale heights from the galaxy plane.

Since the sample galaxies span the entire range of spiral galaxy types and because they were selected on basis of their apparent optical diameters (and thus they also differ greatly in physical sizes), the fact that we find that the vertical scale parameters for both the thin and the thick-disk components are confined to such narrow ranges cannot be explained by invoking selection effects. 
Table 5. Most representative light distributions.

Columns: (1) Galaxy name; (2) Broad-band filter used; (3) Results of vertical model fits; (4) Vertical parameter(s) for best-fitting models (mean parameters as derived from the fits); in case of two-component fits, [1] and [2] indicate the vertical scale parameters for the thin and thick disks, respectively; (5) the scalelengths determined in the exponential regime; (6) the disk scalelengths resulting from a bulge-disk decomposition, and (7) the bulge effective radii, that enclose half the total (bulge) luminosities; and (8) the corresponding reduced $\chi^{2}$ goodness-of-fit parameters for the bulge-disk decompositions, defined as the difference between the observations and the fit scaled to the number of degrees of freedom of the fitted function

\begin{tabular}{|c|c|c|c|c|c|c|c|c|}
\hline $\begin{array}{l}\text { Galaxy } \\
\text { UGC } \\
(1)\end{array}$ & $\begin{array}{l}\text { band } \\
\text { (2) }\end{array}$ & $\begin{array}{c}\text { best model } \\
\text { (3) }\end{array}$ & & $\begin{array}{c}h_{z} \\
\left(h^{-1} \mathrm{kpc}\right) \\
(4)\end{array}$ & $\begin{array}{c}h_{R}^{e x p} \\
\left(h^{-1} \mathrm{kpc}\right) \\
(5)\end{array}$ & $\begin{array}{c}h_{R}^{b / d} \\
\left(h^{-{ }^{-1}} \mathrm{kpc}\right) \\
(6)\end{array}$ & $\begin{array}{c}r_{e} \\
\left(h^{-1} \mathrm{kpc}\right) \\
(7)\end{array}$ & $\begin{array}{c}\text { red. } \chi^{2} \\
(8)\end{array}$ \\
\hline \multirow[t]{4}{*}{7617} & $B$ & sech & & $0.10 \pm 0.03$ & $1.74 \pm 0.08$ & $1.78 \pm 0.11$ & $0.18 \pm 0.02$ & 1.84 \\
\hline & $V$ & $\exp$ & & $0.12 \pm 0.03$ & $1.40 \pm 0.08$ & $1.37 \pm 0.11$ & $0.22 \pm 0.02$ & 1.35 \\
\hline & $R$ & $\exp$ & & $0.11 \pm 0.03$ & $1.29 \pm 0.09$ & $1.37 \pm 0.05$ & $0.27 \pm 0.02$ & 1.64 \\
\hline & $I$ & $\exp$ & & $0.10 \pm 0.03$ & $1.13 \pm 0.08$ & $1.36 \pm 0.11$ & $0.27 \pm 0.02$ & 1.62 \\
\hline \multirow[t]{3}{*}{7808} & $B$ & sech & & $0.12 \pm 0.03$ & $2.72 \pm 0.12$ & $2.69 \pm 0.22$ & $0.39 \pm 0.02$ & 1.38 \\
\hline & $V$ & $\exp$ & & $0.16 \pm 0.04$ & $2.57 \pm 0.12$ & $2.53 \pm 0.15$ & $0.35 \pm 0.02$ & 1.53 \\
\hline & $I$ & $\exp$ & & $0.16 \pm 0.04$ & $1.91 \pm 0.11$ & $2.21 \pm 0.17$ & $0.30 \pm 0.02$ & 1.61 \\
\hline \multirow[t]{4}{*}{$7980^{(1)}$} & $B$ & $\operatorname{sech}+\exp$ & $\begin{array}{l}{[1]} \\
{[2]}\end{array}$ & $\begin{array}{l}0.19 \pm 0.05 \\
0.52 \pm 0.09\end{array}$ & $1.6 \pm 0.1$ & - & & \\
\hline & $V$ & $\operatorname{sech}^{2}+\exp$ & {$[1]$} & $\begin{array}{l}0.17 \pm 0.05 \\
0.47 \pm 0.08\end{array}$ & $1.6 \pm 0.1$ & - & & \\
\hline & $R$ & $\operatorname{sech}+\exp$ & {$[1]$} & $\begin{array}{l}0.15 \pm 0.04 \\
0.48 \pm 0.08\end{array}$ & $1.5 \pm 0.1$ & - & & \\
\hline & $I$ & $\operatorname{sech}^{2}+\exp$ & {$[1]$} & $\begin{array}{l}0.21 \pm 0.05 \\
0.53 \pm 0.09\end{array}$ & $1.4 \pm 0.1$ & - & & \\
\hline \multirow[t]{4}{*}{8016} & $B$ & sech $+\exp$ & {$[1]$} & $\begin{array}{l}0.14 \pm 0.04 \\
0.70 \pm 0.12\end{array}$ & $2.31 \pm 0.07$ & $1.81 \pm 0.09$ & $0.19 \pm 0.02$ & 2.75 \\
\hline & $V$ & $\operatorname{sech}+\exp$ & {$[1]$} & $\begin{array}{l}0.13 \pm 0.03 \\
0.70 \pm 0.11\end{array}$ & $2.24 \pm 0.14$ & $1.78 \pm 0.22$ & $0.21 \pm 0.02$ & 3.93 \\
\hline & $R$ & $\operatorname{sech}+\exp$ & {$[1]$} & $\begin{array}{l}0.13 \pm 0.03 \\
0.60 \pm 0.10\end{array}$ & $2.19 \pm 0.09$ & $1.65 \pm 0.17$ & $0.18 \pm 0.02$ & 3.53 \\
\hline & $I$ & sech $+\exp$ & {$[1]$} & $\begin{array}{l}0.14 \pm 0.04 \\
0.62 \pm 0.10\end{array}$ & $1.91 \pm 0.11$ & $1.42 \pm 0.05$ & $0.15 \pm 0.02$ & 1.73 \\
\hline $8286^{(2)}$ & $\begin{array}{l}B \\
V \\
I\end{array}$ & $\begin{array}{l}\text { sech } \\
\text { sech } \\
\text { sech }\end{array}$ & & $\begin{array}{l}0.12 \pm 0.04 \\
0.13 \pm 0.04 \\
0.13 \pm 0.04\end{array}$ & $\begin{array}{l}2.19 \pm 0.04 \\
2.22 \pm 0.10\end{array}$ & $\begin{array}{l}- \\
-\end{array}$ & & \\
\hline \multirow[t]{3}{*}{8935} & $B$ & sech & & $0.55 \pm 0.09$ & $2.37 \pm 0.09$ & $3.95 \pm 0.26$ & $1.12 \pm 0.05$ & 2.50 \\
\hline & $V$ & $\exp$ & & $0.73 \pm 0.12$ & $2.21 \pm 0.07$ & $3.80 \pm 0.22$ & $1.22 \pm 0.05$ & 2.64 \\
\hline & $I$ & $\exp$ & & $0.79 \pm 0.13$ & $2.14 \pm 0.07$ & $3.60 \pm 0.11$ & $1.30 \pm 0.05$ & 2.24 \\
\hline \multirow[t]{3}{*}{9127} & $B$ & sech & & $0.67 \pm 0.12$ & $3.22 \pm 0.18$ & $4.14 \pm 0.70$ & $1.48 \pm 0.05$ & 1.97 \\
\hline & $V$ & sech & & $0.62 \pm 0.11$ & $2.97 \pm 0.21$ & $3.78 \pm 0.22$ & $1.36 \pm 0.05$ & 2.80 \\
\hline & $I$ & sech & & $0.58 \pm 0.11$ & $2.42 \pm 0.15$ & $3.81 \pm 0.29$ & $1.36 \pm 0.05$ & 2.83 \\
\hline \multirow[t]{3}{*}{10043} & $B$ & sech & & $0.32 \pm 0.09$ & $2.24 \pm 0.16$ & $2.27 \pm 0.55$ & $0.47 \pm 0.03$ & 1.53 \\
\hline & $V$ & $\exp$ & & $0.35 \pm 0.10$ & $1.83 \pm 0.15$ & $2.18 \pm 0.33$ & $0.42 \pm 0.03$ & 1.34 \\
\hline & $I$ & sech & & $0.25 \pm 0.05$ & $1.98 \pm 0.06$ & $2.21 \pm 0.62$ & $0.44 \pm 0.03$ & 1.30 \\
\hline
\end{tabular}

(1) Because of the complicated structure of the central region of UGC 7980, an unambiguous bulge-disk decomposition could not be done. (2) UGC 8286 does not have a bulge component; therefore we fitted various dust models to the disk radial light distribution instead of a bulge-disk decomposition.

\subsection{Radial surface brightness distributions}

\subsubsection{Exponential scalelengths as a function of $z$-height}

If one is interested in the dependence of the exponential radial scale parameter as a function of $z$-height above a galaxy plane, a good first approximation can be obtained by fitting an exponential model to the radial light distributions.

As has been argued by, e.g., Knapen \& van der Kruit (1991), the scale lengths of a particular galaxy, determined by different authors, vary by $\approx 20 \%$. The resulting scale lengths depend heavily on the radial fitting range used, since in general the radial profiles are not exactly expo- nential. Therefore, in order to compare our scale lengths at different passbands, the radial regions over which the disk exponential scale lengths were fitted were chosen in a consistent way.

In order to avoid contaminating bulge light and the possible effects of disk truncations and warps, the fits were done in those (intermediate) regions that are dominated by the disk light, i.e. at those positions where the bulge light contribution is less than $5 \%$ of the total light (see Table 2). Since the scale lengths in $B$ are generally longer than in $I$ and for most of our galaxies the bulge effective radius is also smaller at shorter wavelengths, we used a $B$-band bulge-disk decomposition to define the smallest 


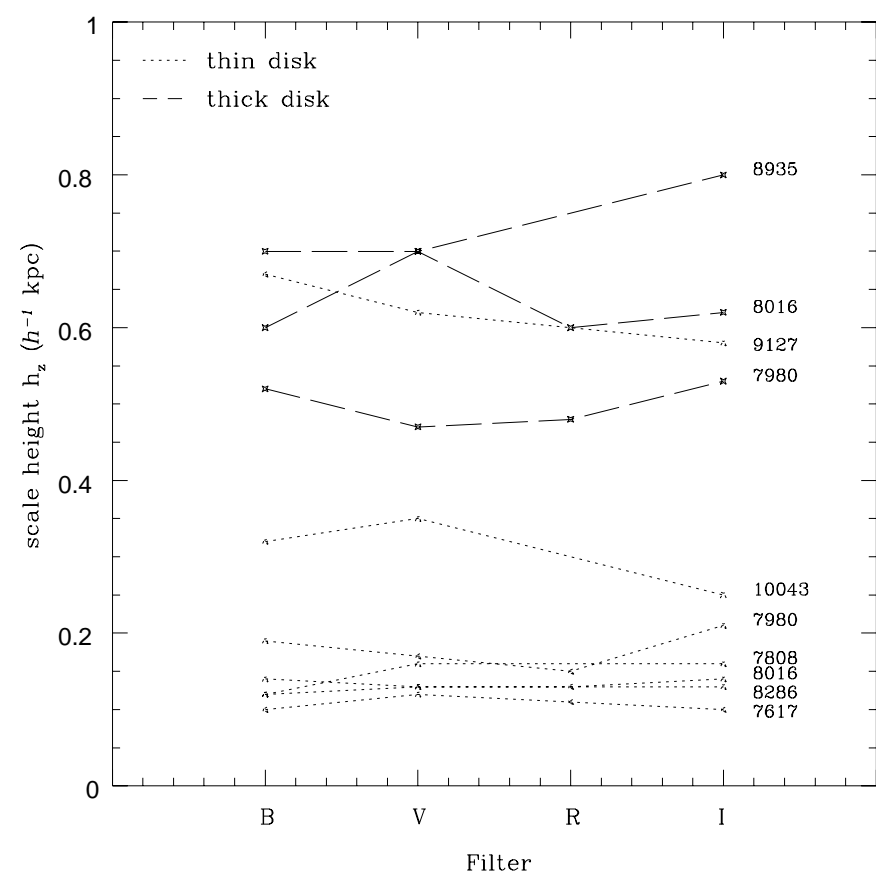

Fig. 4. Scale height distributions for the sample galaxies as a function of passband; the galaxies are indicated by their UGC names

radii of the fitting region at which the bulges contribute less than $5 \%$ for the radial profiles taken furthest from the galaxy planes. The same region was used for all passbands and at all distances from the galaxy planes. The results of these fits are presented in Table 5.

In general the exponential scalelengths decrease with increasing wavelength. This is in agreement with various other studies, e.g. Peletier et al. (1994) find that the scale length in $B$ is usually between 1.2 and 2 times the scale length in $K$ in a representative sample of $\mathrm{Sb}$ and $\mathrm{Sc}$ galaxies. This corresponds to a $B / I$ scale length ratio of about 1.1 to 1.5 (Peletier, priv. comm.) The mean $B$ to $I$ scale length ratio we find here is $1.17 \pm 0.10$, which is within the range found by Peletier et al. (1994). Such a large difference between the scale lengths in these passbands indicates substantial colour gradients in the galaxy disks. It is argued that the ratio depends heavily on the dustiness of the galaxy, e.g. for the early-type galaxies of Balcells \& Peletier (1994) without much visible dust the $B / I$ scale length ratio was found to be $1.04 \pm 0.05$. A similar trend was found by de Jong (1995), who studied a sample of 86 face-on disk-dominated spiral galaxies. He found that the mean $B / K$ scale length ratio of his sample was $1.22 \pm 0.23$. We did not find a clear trend of scale length ratio as a function of galaxy type, large ratios were found in both early and late types. A larger sample is required for a thorough statistical analysis of the behaviour of the scale length ratio as a function of galaxy type.
In all but one of the sample galaxies the scale length decreases when going to longer wavelengths; only in the dusty (late-type) dwarf galaxy UGC 8286 the behaviour is reversed. A complicating factor in this galaxy is the patchiness of the dust distribution, which easily leads to varying estimates of the scale parameter, depending on the way the data is reduced, as was argued by, e.g., Giovanelli et al. (1994). The scalelength distribution as a function of height above the galaxy planes is shown in Fig. 5 for those galaxies with a disk thickness of at least $10^{\prime \prime}$.

\subsubsection{The fitting of more complex models}

We fitted more complex models to the observed light profiles at different $z$-heights, the simplest of these being a bulge-disk decomposition, in which the bulge and the disk are assumed to be the main contributors to the radial light distributions. The advantage of applying a bulge-disk fit to the radial profiles of the sample edge-on galaxies is that we can take into account the bulge influence on the exponentially decreasing disk light distributions. We used a combination of a (spherical) bulge and a disk that are both exponentially-decreasing functions of position along the major axis, rather than the $R^{-1 / 4}$ De Vaucouleurs bulges that are often fitted in the literature. Recent results show that exponential bulges may be even better than the well-known $R^{-1 / 4}$ bulge profiles, depending on galaxy type (e.g., Andredakis \& Sanders 1994; Andredakis et al. 1995; Saraiva Schröder et al. 1994).

Since such decomposition algorithms require very accurate fine-tuning of the bulge and disk parameters, it is extremely difficult to obtain unambiguous results if the observations are rather noisy. In order to determine the radial scalelengths accurately, the fitting should be done in those regions of a galaxy where the influence of a dust layer is minimal, at high $z$-heights. However, at such (relatively) large distances from the galaxy planes, the signal-to-noise ratios are generally rather poor, so the decomposition into bulge and disk parameters is usually not well determined. Therefore, in Table 5 we have listed only those results from the decompositions for which we have integrated the light over all $z$ of the total galaxies (all $R$ ).

Although the influence of a possible dust lane (in the galaxy planes) cannot be quantified here, the scalelengths in Table 5 can be used for a first-order comparison with the scalelengths resulting from the fitting of an exponential light distribution (Sect. 3.2.1); Cols. (5) and (6) illustrate that, in general, they agree on a $20 \%$ level. The difference between these values obtained for the scale lengths is largely due to the difference in fitting boundaries and is thus a measure for the realistic error in $h_{R}$.

A bulge component was not found in only one of the sample galaxies, UGC 8286 . Since extinction dramatically affects fits to radial (disk) profiles we have fitted the radial light distribution of UGC 8286 with extinction-dependent models taken from KB87 (their Eqs. (14-16)). Radial 

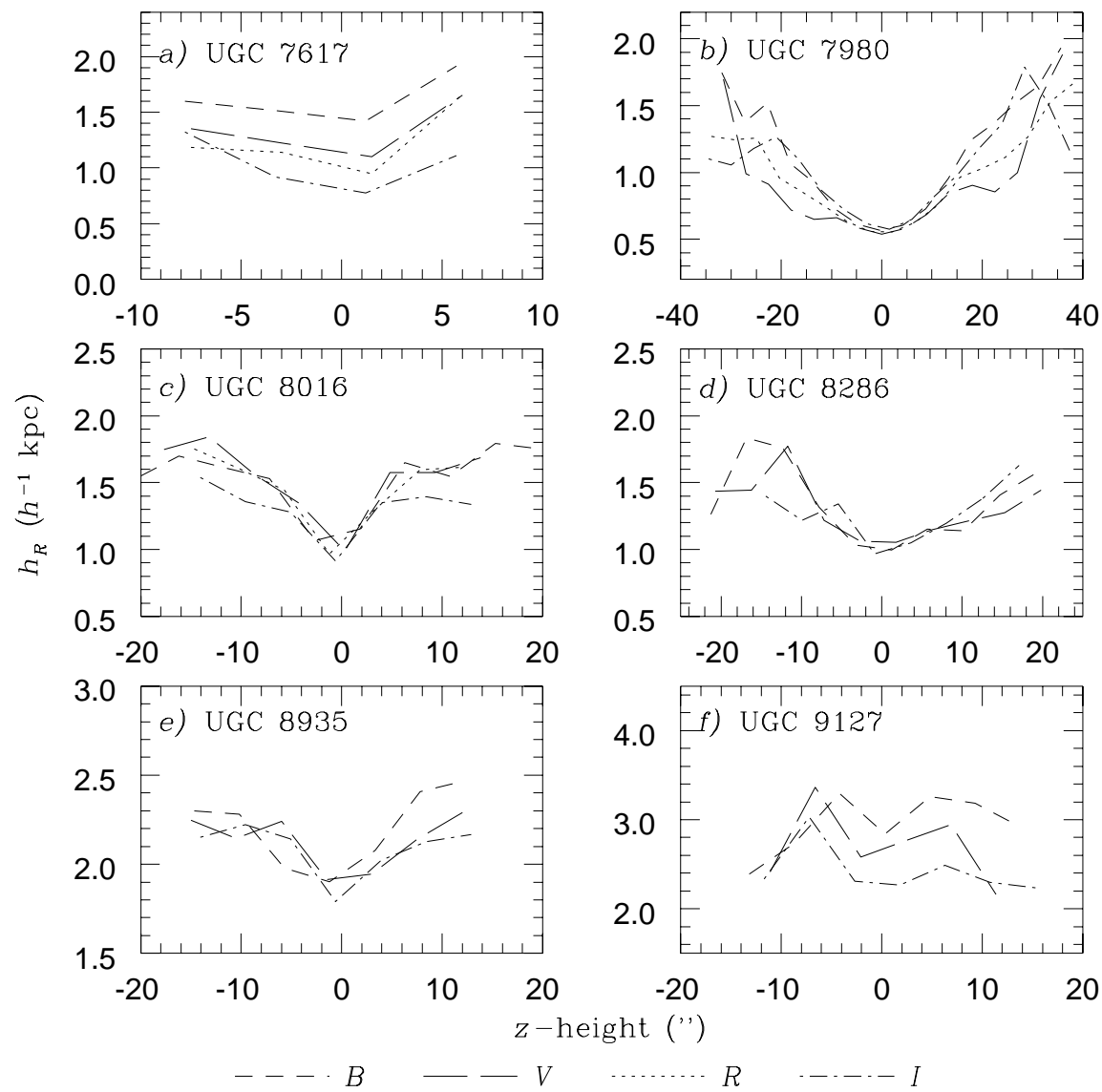

Fig. 5. Exponential scalelength distributions as a function of $z$-height for the sample galaxies with a disk thickness of at least $10^{\prime \prime}$. For clarity the statistical errors are not shown in the panels, indicative error estimates are given in Table 5

profiles at different distances from the galaxy plane were fitted with total extinction (absorption and scattering) coefficients at the galaxy centers, $\kappa_{0}$, of $0.1,0.5,1.0,2.0$, and $5.0 \mathrm{mag}$; the results are shown in Fig. 6, together with the results from fitting purely exponential light distributions to the radial disk profiles. Best fits were obtained with the smallest values for $\kappa_{0},\left(\kappa_{0}(B, V)=0.5, \kappa_{0}(I)=0.1\right)$, with little variation as a function of distance from the galaxy plane. Only a very slight hint of an increasing extinction coefficient towards the galaxy plane was observed. The best-fitting scalelengths, determined at high $z$-heights in order to minimize the influence of a dust lane, are $h_{R, B}=1.20 \pm 0.04 h^{-1} \mathrm{kpc}, h_{R, V}=1.01 \pm 0.07$, and $h_{R, I}=1.4 \pm 0.1$, slightly smaller than those obtained by fitting an exponentially-decreasing luminosity distribution to the outer parts. In contrast, Van der Kruit \& Searle (1982a) found an exponential scalelength of $1.5 h^{-1} \mathrm{kpc}$ for this galaxy, and $2.6 h^{-1} \mathrm{kpc}$ if only the inner $2.3 \mathrm{kpc}$ are considered, in the broad-band optical colours $U^{\prime}, J$, and $F$. The large discrepancy between the various values found here shows that this kind of modeling only yields scale lengths accurate to $\sim 50 \%$, but it might be useful to measure variations of scale length as a function of height above the plane.

DDP found from their model calculations that if real galaxies can be represented by sandwich models, which would seem to be the case from observations of edge-on galaxies (e.g. KB87), then no matter how large their optical depths, they will behave in the optical as if they are optically thin. Our results support this conclusion.

Jensen \& Thuan (1982) have examined the behaviour of the scalelength at different heights above the plane of the edge-on NGC 4565 and found the scalelengths to be rather constant in those regions where the influence of dust and young stars is considered to be negligible (with variations on order of 1\%). Therefore they concluded that the scaleheight is independent of radius. We can see from Fig. 5 that a number of galaxies in our sample have a scalelength distribution that is more or less flat at those $z$ heights where the dust influence is assumed to be minimal. If we combine this with the results obtained in Sects. 3.1.1 and 3.1.2, we see that, except for early-type galaxies, the scale height distribution is rather constant with height above the plane. 
Table 6. Positions along the major axes and widths (in arcseconds) of the extracted profiles. The first profiles refer to the top ones in Fig. 3; the galaxy centers are at position 0

\begin{tabular}{|c|c|c|c|c|c|c|c|c|c|c|c|c|c|c|c|c|}
\hline \multirow[b]{2}{*}{ nr. } & \multicolumn{2}{|c|}{ UGC 7617} & \multicolumn{2}{|c|}{ UGC 7808} & \multicolumn{2}{|c|}{ UGC 7980} & \multicolumn{2}{|c|}{ UGC 8016} & \multicolumn{2}{|c|}{ UGC 8286} & \multicolumn{2}{|c|}{ UGC 8935} & \multicolumn{2}{|c|}{ UGC 9127} & \multicolumn{2}{|c|}{ UGC 10043} \\
\hline & pos. & width & pos. & width & pos. & width & pos. & width & pos. & width & pos. & width & pos. & width & pos. & width \\
\hline 1 & -20 & 8 & -40 & 30 & -90 & 15 & -60 & 28 & -70 & 15 & -40 & 28 & -85 & 28 & -60 & 15 \\
\hline 2 & -15 & 8 & -6 & 8 & -70 & 8 & -40 & 15 & -60 & 15 & -20 & 15 & -65 & 15 & -50 & 15 \\
\hline 3 & -10 & 8 & -3 & 3 & -35 & 8 & -25 & 15 & -50 & 8 & -10 & 8 & -50 & 15 & -40 & 8 \\
\hline 4 & -5 & 8 & 2 & 3 & -20 & 8 & -15 & 8 & -20 & 8 & 10 & 8 & -40 & 8 & -20 & 8 \\
\hline 5 & 5 & 8 & 4 & 8 & -10 & 8 & -5 & 3 & -10 & 3 & 20 & 15 & -35 & 8 & 25 & 8 \\
\hline 6 & 8 & 8 & 10 & 8 & 10 & 3 & 20 & 8 & -5 & 3 & 40 & 28 & 20 & 8 & 30 & 8 \\
\hline 7 & 12 & 8 & 20 & 15 & 20 & 8 & 30 & 15 & 10 & 3 & & & 40 & 8 & 45 & 8 \\
\hline 8 & 22 & 15 & 40 & 28 & 35 & 8 & 50 & 28 & 20 & 3 & & & 60 & 15 & 60 & 15 \\
\hline 9 & & & & & 70 & 15 & & & 50 & 8 & & & 75 & 15 & & 15 \\
\hline 10 & & & & & 90 & 28 & & & 60 & 8 & & & 95 & 28 & & \\
\hline
\end{tabular}

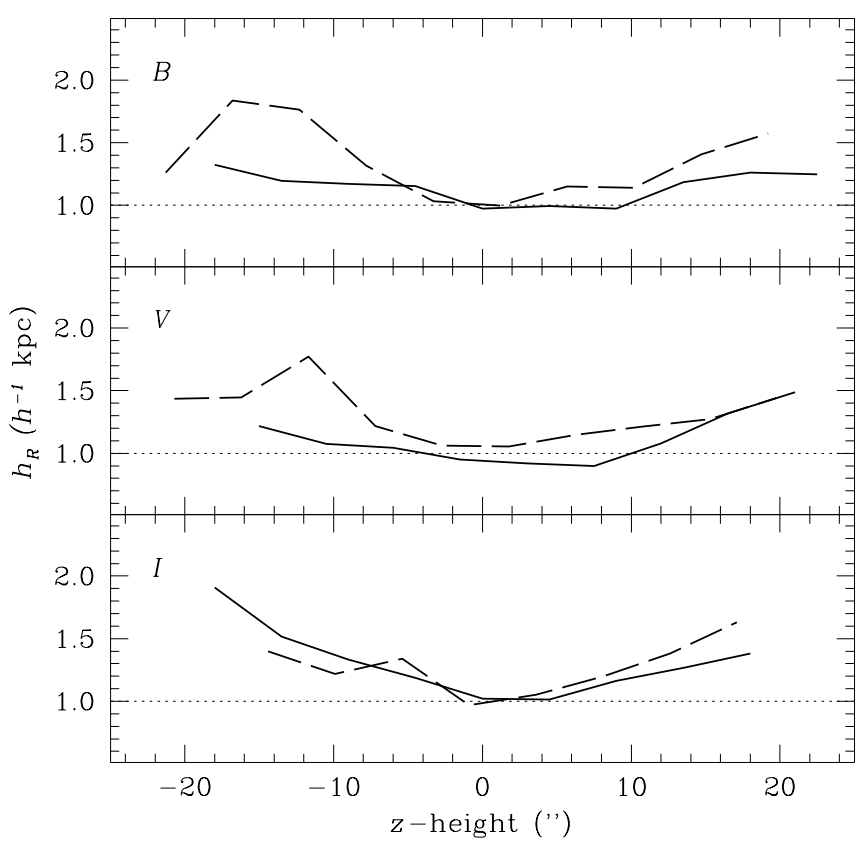

Fig. 6. Scalelength distribution as a function of distance from the galaxy plane of UGC 8286. The solid lines represent the distributions resulting from the extinction-dependent modeling, the dashed lines were derived from the simple exponential fits. We see a strong disagreement between the two distributions south of the galaxy plane, due to the dustiness of UGC 8286. The dotted line is meant to guide the eye

\subsubsection{The ratio of radial to vertical scale parameters}

Since we are looking at edge-on galaxies, we can easily measure the ratio of the radial to the vertical scale parameter, $h_{R} / z_{0}$. So far, this ratio is only known for a few galaxies.

However, when studying edge-on galaxies we are looking at the projected disk light, integrated along the line of sight. In other words, the resulting exponential radial scale parameters will differ systematically from the true exponential scalelengths. As has been shown in the previous
Section, we may assume that the maximum extinction coefficient $\kappa_{0}$ will be on order unity. Under this assumption, the systematic differences between the true exponential scalelengths and the exponential scale parameters found are on order of $10 \%$ at maximum. So the values for the radial scale parameters found may have to be increased by up to $10 \%$, depending on the amount of extinction encountered in the individual galaxies.

In Table 7 we have listed the $h_{R} / z_{0}$ ratios as found for the sample galaxies. $I$-band parameters are used, because in $I$ the influence of dust extinction is the smallest of the optical bands. The mean $h_{R} / z_{0}$ ratio of this sample is 5.6 \pm 0.4 , where the errors are $1 \sigma$ statistical errors. If we leave out the scale parameter ratio for UGC 9127, for which the scale height was shown to be rather large compared to the scale height of the other sample galaxies, we find a mean $h_{R} / z_{0}$ ratio of $5.9 \pm 0.4$.

\section{Discussion}

\subsection{Main observational results}

As shown in the previous section, our main observational results are the following:

- Especially in the later-type galaxies, the scale height was found to be constant as a function of position along the major axis; the constancy is $\sim 10-15 \%$ in galaxies of type Sc and later.

- In most of the sample galaxies the scale heights of the vertical luminosity profiles seem to increase slightly when going to larger distances from the galaxy centers.

- In the later-type galaxies (Sc and later) the vertical light distributions are best represented by either an exponential or a $\operatorname{sech}(z)$ single component model; in the early-type galaxies an underlying thick disk was found.

- The range of vertical scale parameters is quite narrow for both the thin and the thick disk components; no clear variations as a function of passband were found. 
- The exponential scale lengths decrease with increasing wavelength, and also with distance from the galaxy planes

- The mean $h_{R} / z_{0}$ ratio of this sample is $5.9 \pm 0.4$.

Table 7. Scale parameter ratios

\begin{tabular}{lcccc}
\hline \hline Galaxy & $\left(h_{R} / z_{0}\right)_{B}$ & $\left(h_{R} / z_{0}\right)_{V}$ & $\left(h_{R} / z_{0}\right)_{R}$ & $\left(h_{R} / z_{0}\right)_{I}$ \\
\hline UGC 7617 & 8.90 & 5.71 & 6.23 & 6.80 \\
UGC 7808 & 11.2 & 7.91 & - & 6.91 \\
UGC 7980 & 4.2 & 4.7 & 5.0 & 3.3 \\
UGC 8016 & 6.46 & 6.85 & 6.35 & 5.07 \\
UGC 8286 & 9.12 & 8.53 & - & 11.8 \\
UGC 8935 & $3.59(6.6)$ & $2.60(4.8)$ & - & $2.28(4.5)$ \\
UGC 9127 & 3.09 & 3.05 & - & 4.42 \\
UGC 10043 & 3.55 & 3.11 & - & 4.42 \\
\hline
\end{tabular}

The scalelengths used for this table are those measured in the regions that were minimally influenced by dust. For UGC 8935 the ratios in between brackets are those derived from the thick/thin disk decomposition of Sect. 3.1.4.

\subsection{The vertical scale parameter}

As has been shown in Sect. 3.1.1, the exponential disk scale height seems to be (more or less) independent of position along the major axis for the galaxies in the present sample, the scale height variations in the later-type galaxies were found to be of order 10 to $15 \%$ at maximum. As was noted in Sect. 3.1.1, however, the scale height seems to increase in the outer parts of the galaxies. It is therefore possible that the disk thickens just before the optical edge (van der Kruit \& Searle 1981a,b, 1982a,b).

However, even if the shallowing at the last profiles were entirely due to a thickening of the disk, the implied change in $z_{0}$ would be relatively small and restricted to no more than $10 \%$ of the obervable extent of the galaxies (van der Kruit \& Searle 1981a). So it is a good approximation to take the vertical scale parameter independent of position along the major axis, because except for these outermost profiles the models fit the observations very well.

Another possibility is that optical warps are present, like in the HI, causing the shallower outer profiles (van der Kruit 1979).

In Sect. 3.1.2 we derived the best-fitting vertical scale parameters $h_{z}$ for the main disk components. As we already noted, these are in the range of 100 to $300 \mathrm{pc}$ for late-type galaxies and the thin disks of early type galaxies. If we compare these values with the values derived for other galaxies, then we find that they are comparable. For instance, for the Galaxy, Gilmore \& Reid (1983) and others find a scale height $h_{z}=325 \pm 25$ pc for the old-disk stars. Comparable values for other edge-on spiral galaxies were found by, e.g., van der Kruit \& Searle (1981a,b; 1982a,b) and Hamabe (1982). By using $2.4 \mu \mathrm{m}$ data, Kent et al. (1991) found a scale height for the Galaxy in the solar neighbourhood of $h_{z} \sim 247 \mathrm{pc}$ (assuming $R_{\odot}=8 \mathrm{kpc}$ ), decreasing to $h_{z} \sim 165 \mathrm{pc}$ at $R=5 \mathrm{kpc}$.
Finally, we did not detect a clear variation of the scale height with passband. However, in the presence of a dust layer and different stellar populations, such variations would be expected: in general, the older population has a greater velocity dispersion and therefore a larger scale height than the young population. In the $B$ band, the main contributor to the galaxy light is the young population, but a large fraction of its light is obscured by the dust component, yielding too small values for the exponential scale heights. In $V$, the contribution of the young population is small compared to that of the older (and redder) population, whereas the dust still plays an important role. Only in $R$ and $I$ the dust influence is much smaller and the older population shows up more clearly.

\subsection{The dust contribution}

As was noted in the previous section, we would expect a variation of scale height with wavelength in the presence of a dust lane and different stellar populations. However, in general such a variation was not detected. Also, we have shown that the scale lengths vary as a function of $z$ distance from the galaxy planes. The smallest scale lengths were measured in the galaxy planes.

A possible explanation for the absence of a significant variation in scale height as a function of passband is that, even in the $I$ band, the entire galaxy is completely optically thick (in the regions fitted). If the edge-on opacity of galaxy disks is much greater than unity, then the observed light distributions will give rise to more or less identical (radial and vertical) slopes. However, if this were the case, we would also expect a flat distribution of scale lengths as a function of distance from the galaxy planes.

On the other hand, we could explain both observational facts by assuming that the region in which the opacity is much greater than unity is confined to a narrow strip close to the galaxy planes and increasingly important towards the galaxy centers. In that case, we assume that the light distributions we observe represent the same stellar population in the optical passbands observed in, and are only very slightly affected by absorption effects. The smaller scale lengths close to the galaxy planes can then be explained by a combination of absorption effects and a young stellar population, that is supposed to be present in the galaxy planes.

In terms of scale lengths ratios, Peletier et al. (1994) have come up with a similar result. They have shown that many of their sample $\mathrm{Sb}$ and $\mathrm{Sc}$ galaxies that are highly inclined have small scale length ratios and as such cannot contain a lot of dust away from the galaxy planes.

As has been shown by, e.g., Giovanelli et al. (1994) and Knapen \& van der Kruit (1991), the photometric profiles will yield different values for the scale parameters, depending on the dust distribution and the ranges assumed for the fitting of the scale parameters. The effect of absorption, concentrated towards the profiles' 
centers, is to increase the scale parameter values, and hence the scale parameters tend to be overestimated at smaller wavelengths. Therefore, the ratio of $B$ to $I$-band scale parameters gives an indication of the influence of absorption in galaxies, as was already mentioned in Sect. 3.2.1. However, such a global comparison will break down when analyzing individual galaxies, since the assumption of a smoothly, uniformly distributed dust layer does not hold on scales smaller than the galaxies, due to the patchiness of the dust distribution.

Although we used regions away from the central planes of the galaxies, in order to avoid the contamination of dust and stellar light, even at these $z$ distances the observed light distributions consist of a (nonuniform) mixture of stars and dust (though less than in the planes).

\subsection{Disk components}

What we learn from studying the $I$-band data, where the influence of an obscuring dust layer is minimal, is that the model that represents the vertical light distribution best is either an exponential or a $\operatorname{sech}(z)$ distribution. We need, however, near-infrared observations to distinguish between both models, because only then we expect to obtain images that are not influenced by dust extinction. By supplementing the data presented here with that of Wainscoat et al. (1989), who obtained near-infrared observations of the southern edge-on galaxy IC 2531, we may argue that the vertical stellar distribution is generally exponential. However, we should be careful when interpretating Wainscoat et al.'s (1989) data, because of their poorer resolution: in the inner parts they cannot distinguish between exponential and $\operatorname{sech}(z)$-distributions. Barnaby \& Thronson Jr. (1992) have studied the vertical disk light distribution of the late-type edge-on NGC 5907 in the near-infrared $H$-band. They found, following van der Kruit's (1988) suggestion, that they could model the vertical disk profiles with a $\operatorname{sech}(z)$ function. However, they did not try to fit their data with an exponential light distribution. Better data on these galaxies is available now, both in the optical and in the near-infrared. We will publish the results obtained from those data in a subsequent paper.

As was noted in Sect. 3.1.2 already, the vertical light distributions of the early-type galaxies in our sample are best represented by two-component models. This second component was first detected by Burstein (1979) in a sample of edge-on S0 galaxies; he called it a "thick disk" because of the larger scale height than that of the dominant component. A number of possible origins for these thick disks can be thought of. They could be truly separate dynamical components of the galaxies, or due to the bulge light contribution, as was suggested by Freeman (1985). However, Shaw \& Gilmore (1989) did not find any evidence for a significant bulge light contribution to the thick-disk light of NGC 891, even though they could study its photometric data to distances from the galaxy plane of $4.5 \mathrm{kpc}$ at major axis distances of $15 \mathrm{kpc}$ (assuming a distance of $9.5 \mathrm{Mpc}$, following van der Kruit \& Searle 1981b) and $\sim 8 \mathrm{kpc}$ down the minor axis.

Although the presence of a thick disk component in our Galaxy is generally accepted (having a scale height of $\sim 1$ $\mathrm{kpc}$ ), in external galaxies, the existence of a thick disk has not been proven unambiguously. The profiles of NGC 891 can be explained by a thick disk, but also by a "returning" bulge with axis ratios increasing towards the outer parts (van der Kruit 1984; Bahcall \& Kylafis 1985). Probably one of the most convincing examples of the existence of a thick disk component was found by van Dokkum et al. (1994) in the case of the edge-on spiral NGC 6504, having a scale height of approximately 4 times the thin-disk scale height.

The ratios of the scale heights of the two components found for UGC 7980 and UGC 8016 are $2.8 \pm 0.2$ and $5.0 \pm 0.4$, respectively. If we want to compare these values to those found for other galaxies, only a few references are available. Van der Kruit (1987a) found ratios of 3.2 and 4.0 for NGC 891 and the Galaxy, respectively. Jensen \& Thuan (1982) derived a somewhat larger ratio of 6.6 for the edge-on Sc galaxy NGC 4565. Furthermore, as van Dokkum et al. (1994) have shown, for the edge-on Sab galaxy NGC 6504 a ratio of about 4 was found. So the ratios found in this study are consistent with data from the literature.

We have looked for the existence of possible thick disks in the other sample galaxies as well, but no such components were found.

In this paper we have shown that the mean ( $I$-band) scale parameter ratio is $5.9 \pm 0.4$, which agrees very well with the value found by van der Kruit \& Searle (1982a). A number of studies have dealt with the determination of the $h_{R} / z_{0}$ ratio for our Galaxy, including van der Kruit (1987b) who finds $\left(h_{R} / z_{0}\right)_{\text {Gal }}=8.5 \pm 1.3$, closer to the theoretical value of about 10 needed for disks of Sc galaxies with maximum rotation (Bottema 1993).

\section{Summary and conclusions}

In this paper we have tried to determine the structural parameters of a small sample of extremely inclined spiral galaxies.

- Vertically, we have distinguished between a simple exponential light distribution and the isothermal $\operatorname{sech}^{2}(z)$ profile. In general, the simple exponential fits turned out to be good approximations of the stellar light distribution, although more complicated model fitting was required for a detailed description of the disk components.

- The exponential parts of the vertical profiles were used to study the vertical scale parameter as a function of position along the major axis. It was found that the 
disk scale height is constant with radius, although the constancy seems to lose strength at larger radii: there is a hint of a radial increase of the exponential scale height.

- Neither the isothermal nor the exponential model could account for all profiles observed, so an intermediate $\operatorname{sech}(z)$ model had to be invoked, as was also proposed by van der Kruit (1988). In most cases, either the exponential or the $\operatorname{sech}(z)$ yields good fits to the data, thus leading to the conclusion that in the sample galaxies, at least near the plane, we are not looking at one isothermal population, but probably at a mix of several stellar populations as well as a dust component. The $\operatorname{sech}^{2}(z)$ distributions generally fit worse.

- Deviations from an exponential or $\operatorname{sech}(z)$ model can be accounted for by invoking the influence of dust or a young stellar population near the equatorial planes of the galaxies, whereas the excess of light that is detected in a few early-type galaxies is possibly due to the presence of an underlying thick disk component. The relationship between the presence of thick disks and bulges will be studied in future papers for a larger, statistically complete sample of edge-on spiral galaxies.

- It was found that the scale heights of both the main (or thin) disk component and of the thick disk component (if present) are confined within narrow ranges.

- By studying the scalelength variations at different $z$ heights above the galaxy planes one can get an idea of the influence of dust and a young stellar population on the optical emission. From the observations we conclude that their influence is to decrease the scalelengths towards the galaxy planes. Under this assumption, the implication then is that by taking the in-plane scalelengths we underestimate the true scalelengths of the light and possibly of the main mass component as well. This may have severe implications on rotation curve fitting and the dark matter problem.

- We fitted different dust models to the only sample galaxy without a bulge, UGC 8286, assuming equal scalelengths for the dust and the stars. The resulting total extinction coefficients lie in the optically thin regime. This supports the assumption that dust in galaxies is generally distributed in a flat layer near the galaxy planes.

- The ratio of the radial and vertical scale parameters, $h_{R} / z_{0}$, was found to be $5.9 \pm 0.4$ in the $I$ band, which is less influenced by extinction effects than other optical bands. The values for $h_{R}$ were calculated for the integrated light at those distances above the galaxy planes where the influence of the central dust lane (if present) was minimal. This ratio, although comparable to van der Kruit \& Searle's (1982a) scale parameter ratio, is too small to account for maximum rotation in a galaxy disk (Bottema 1993).
Acknowledgements. We would like to thank Reynier Peletier, Penny Sackett, Roelof de Jong, Yiannis Andredakis, Roelof Bottema, Rob Swaters and Erwin de Blok for the many useful discussions and suggestions concerning this work, and Kor Begeman for his software support. This paper is based on data from the Jacobus Kapteyn Telescope at La Palma, operated by the Royal Greenwich Observatory at the Observatorio del Roque de los Muchachos of the Instituto de Astrofísica de Canarias, with financial support of PPARC (UK) and NWO (NL).

\section{References}

Andredakis Y.C., Peletier R.F., Balcells M., 1995, MNRAS (in press)

Andredakis Y.C., Sanders R.H., 1994, MNRAS 267, 283

Bahcall J.N., Casertano S., 1984, ApJ 284, L35

Bahcall J.N., Kylafis N.D., 1985, ApJ 288, 252

Balcells M., Peletier R.F., 1994, AJ 107, 135

Barnaby D., Thronson Jr. H.A., 1992, AJ 103, 41

Bertin G., Lin C.C., 1987, in: Palous, J. (ed.), Evolution of Galaxies; Pub. Astr. Inst. Czechoslovakian Acad. Sci. 69, 255

Bottema R., 1993, A\&A 275, 16

Burstein D., 1979, ApJ 234, 829

Carlberg R.G., Sellwood J.A., 1985, ApJ 292, 79

Cowie L.L., Songaila A., 1986, Ann. Rev. A\&A 24, 499

de Jong R.S., 1995, Ph.D. Thesis, University of Groningen

de Vaucouleurs G., de Vaucouleurs A., Corwin H.G. Jr., Buta R.J., Paturel G., Fouqué P., 1991. Springer-Verlag, New York

Disney M., Davies J., Phillipps S., 1989, MNRAS 239, 939 (DDP)

Freeman K.C., 1970, ApJ 160, 811

Freeman K.C., 1978, in: Berkhuijsen \& Wielebinski (eds.) IAU Symposium 77, p. 3

Freeman K.C., 1985, in "The Milky Way Galaxy", IAU Symposium 106 In: van Woerden H. et al. (eds.), p. 113

Gilmore G., Reid N., 1983, MNRAS 202, 1022

Giovanelli R., Haynes M.P., Salzer J.J., Wegner G., Da Costa L.N., Freudling W., 1994, AJ 107, 2036

Guthrie B.N.G., 1992, A\&AS 93, 255

Hamabe M., 1982, PASJ 34, 423

Hubble E., 1926, ApJ 64, 321

Jenkins A., 1992, MNRAS 257, 620

Jensen E.B., Thuan T.X., 1982, ApJS 50, 421

Kent S.M., Dame T.M., Fazio G., 1991, ApJ 378, 131

Knapen J.H., van der Kruit P.C., 1991, A\&A 248, 57

Kylafis N.D., Bahcall J.N., 1987, ApJ 317, 637

Lacey C.G., 1984, MNRAS 208, 687

Landolt A.U., 1983, AJ 88, 439

Landolt A.U., 1992, AJ 104, 340

Marquardt J., 1963, J. Soc. Ind. Appl. Math. 11, 431

Nilson P., 1973, Uppsala General Catalogue of Galaxies (UGC), Uppsala

Peletier R.F., Valentijn E.A., Moorwood A.F.M., Freudling W., 1994, A\&AS 108, 621

RGO/La Palma Technical Notes No. 37, 1987 (Isaac Newton Group, La Palma)

RGO/La Palma Technical Notes No. 73, 1990 (Isaac Newton Group, La Palma) 
Russell J.L., Lasker B.M., McLean B.J., Sturch C.R., Jenkner H., 1990, AJ 99, 2059

Saraiva Schröder M.F., Pastoriza M.G., Kepler S.O., Puerari I., A\&AS 108, 41

Shaw M.A., Gilmore G., 1989, MNRAS 237, 903

Shaw M.A., Gilmore G., 1990, MNRAS 242, 59

Spitzer L., Schwarzschild M., 1951, ApJ 114, 385

Toomre A., 1964, ApJ 139, 1217

Tsikoudi V., 1977, Univ. Texas Publ. Astron. No. 10

van der Kruit P.C., 1979, A\&AS 38, 15

van der Kruit P.C., 1984, A\&A 140, 470

van der Kruit P.C., 1987a, A\&A 173, 59

van der Kruit P.C., 1987b, in "The Galaxy". In: Gilmore \&
Carswell, p. 17

van der Kruit P.C., 1988, A\&A 192, 117

van der Kruit P.C., Searle L., 1981a, A\&A 95, 105

van der Kruit P.C., Searle L., 1981b, A\&A 95, 116

van der Kruit P.C., Searle L., 1982a, A\&A 110, 61

van der Kruit P.C., Searle L., 1982b, A\&A 110, 79

van Dokkum P.G., Peletier R.F., de Grijs R., Balcells M., 1994, A\&A 286, 415

Wainscoat R.J., 1986, Ph.D. Thesis, Australian National University

Wainscoat R.J., Freeman K.C., Hyland A.R., 1989, ApJ 337, 163 